\title{
Regional surface morphology of comet 67P/Churyumov-Gerasimenko from Rosetta/OSIRIS images: The southern hemisphere
}

\author{
M. R. El-Maarry ${ }^{1}$, N. Thomas ${ }^{1}$, A. Gracia-Berná1 ${ }^{1}$ M. Pajola ${ }^{2}$, J.-C. Lee ${ }^{3}$, M. Massironi ${ }^{4}$, B. Davidsson ${ }^{5}$, S. Marchi ${ }^{6}$, \\ H. U. Keller ${ }^{7}$, S. F. Hviid ${ }^{8}$, S. Besse ${ }^{9}$, H. Sierks ${ }^{10}$, C. Barbieri ${ }^{11}$, P. L. Lamy ${ }^{12}$, D. Koschny ${ }^{9}$, H. Rickman ${ }^{14,15}$, \\ R. Rodrigo ${ }^{13,14}$, M. F. A'Hearn ${ }^{17}$, A.-T. Auger ${ }^{12}$, M. A. Barucci ${ }^{18}$, J.-L. Bertaux ${ }^{19}$, I. Bertini ${ }^{20}$, D. Bodewits ${ }^{17}$, \\ G. Cremonese ${ }^{11}$, V. Da Deppo ${ }^{21}$, M. De Cecco $^{22}$, S. Debei ${ }^{23}$, C. Güttler ${ }^{10}$, S. Fornasier ${ }^{19}$, M. Fulle ${ }^{24}$, L. Giacomini ${ }^{4}$, \\ O. Groussin ${ }^{12}$, P. J. Gutierrez ${ }^{25}$, W.-H. Ip ${ }^{26}$, L. Jorda ${ }^{27}$, J. Knollenberg ${ }^{8}$, G. Kovacs ${ }^{10}$, J.-R. Kramm ${ }^{10}$, E. Kührt ${ }^{8}$, \\ M. Küppers ${ }^{28}$, L. M. Lara ${ }^{25}$, M. Lazzarin ${ }^{11}$, J. J. Lopez Moreno ${ }^{25}$, R. Marschall ${ }^{1}$, F. Marzari ${ }^{11}$, G. Naletto ${ }^{20,21,29}$, \\ N. Oklay ${ }^{10}$, A. Pommerol ${ }^{1}$, F. Preusker ${ }^{8}$, F. Scholten ${ }^{8}$, C. Tubiana ${ }^{10}$, and J.-B. Vincent ${ }^{10}$
}

(Affiliations can be found after the references)

Received 4 April 2016 / Accepted 31 May 2016

\begin{abstract}
Aims. The OSIRIS camera on board the Rosetta spacecraft has been acquiring images of the comet 67P/Churyumov-Gerasimenko (67P)'s nucleus since August 2014. Starting in May 2015, the southern hemisphere gradually became illuminated and was imaged for the first time. Here we present the regional morphology of the southern hemisphere, which serves as a companion to an earlier paper that presented the regional morphology of the northern hemisphere.

Methods. We used OSIRIS images that were acquired at orbits $\sim 45-125 \mathrm{~km}$ from the center of the comet (corresponding to spatial resolutions of $\sim 0.8$ to $2.3 \mathrm{~m} / \mathrm{pixel}$ ) coupled with the use of digital terrain models to define the different regions on the surface, and identify structural boundaries accurately.

Results. Seven regions have been defined in the southern hemisphere bringing the total number of defined regions on the surface of the nucleus to 26. These classifications are mainly based on morphological and/or topographic boundaries. The southern hemisphere shows a remarkable dichotomy with its northern counterpart mainly because of the absence of wide-scale smooth terrains, dust coatings and large unambiguous depressions. As a result, the southern hemisphere closely resembles previously identified consolidated regions. An assessment of the overall morphology of comet 67P suggests that the comet's two lobes show surface heterogeneities manifested in different physical/mechanical characteristics, possibly extending to local (i.e., within a single region) scales.
\end{abstract}

Key words. comets: general - comets: individual: 67P/Churyumov-Gerasimenko - methods: observational

\section{Introduction}

The Rosetta mission has been in orbit around comet 67P/Churyumov-Gerasimenko (67P) since August 2014 obtaining very high-resolution images (down to $<20 \mathrm{~cm} / \mathrm{px}$ ) using the Optical, Spectroscopic, and Infrared Remote Imaging System (OSIRIS, Keller et al. 2007), particularly for the comet's northern hemisphere. The northern hemisphere was found to be morphologically diverse (Sierks et al. 2015; Thomas et al. 2015b; El-Maarry et al. 2015a) including regions of consolidated, often fractured (El-Maarry et al. 2015b; Rickman et al. 2015) units, materials of variable strength and cohesion (Groussin et al. 2015a), smooth terrains showing aeolian-like landforms (Thomas et al. 2015b; El-Maarry et al. 2015a) and seasonal variations (Groussin et al. 2015b), dust-covered areas suggestive of an air-fall-like mechanism (Thomas et al. 2015a), and irregular large-scale depressions suggestive of massive outburst activities (El-Maarry et al. 2015a). Associated with these various terrains are a number of interesting features, which include active pits (Vincent et al. 2015), boulders of variable sizes (Pajola et al. 2015), some of which are presumed to be icerich (Pommerol et al. 2015), pits suggestive of fluidized outflow processes (Thomas et al. 2015b; El-Maarry et al. 2015a; Auger et al. 2015), and numerous terraced outcrops suggestive of global layering (Massironi et al. 2015).
A similarly detailed mapping of the comet's southern hemisphere was not possible before the comet's equinox (in May 2015), closely preceding perihelion (in Aug. 2015). However, the increased activity as the comet came closer to perihelion forced ESA to move Rosetta into larger orbits around the nucleus to protect the spacecraft. These cautionary measures, followed with an excursion at $1000 \mathrm{~km}$ away from the nucleus to carry out important scientific investigations of the coma, limited geological investigation of the comet's southern hemisphere. In early December 2015, Rosetta was moved again into close orbits permitting detailed investigations.

Here, we present the regional morphology of the recently illuminated regions of the southern hemisphere as a follow-up and a companion to an earlier paper that presented the regional morphology of the northern hemisphere (El-Maarry et al. 2015a), and we highlight the most remarkable morphological features in each of these southern regions. In total, seven regions have been defined on the southern hemisphere (Fig. 1) which, in addition to the 19 regions identified on the northern hemisphere (see Fig. A.1), bring the total number of distinct regions on the surface of the nucleus to 26 . Finally, we place the new regions in the context of the cometary nucleus as a whole and draw comparisons with the northern hemisphere regions to infer hemispherical differences and their possible implications. 


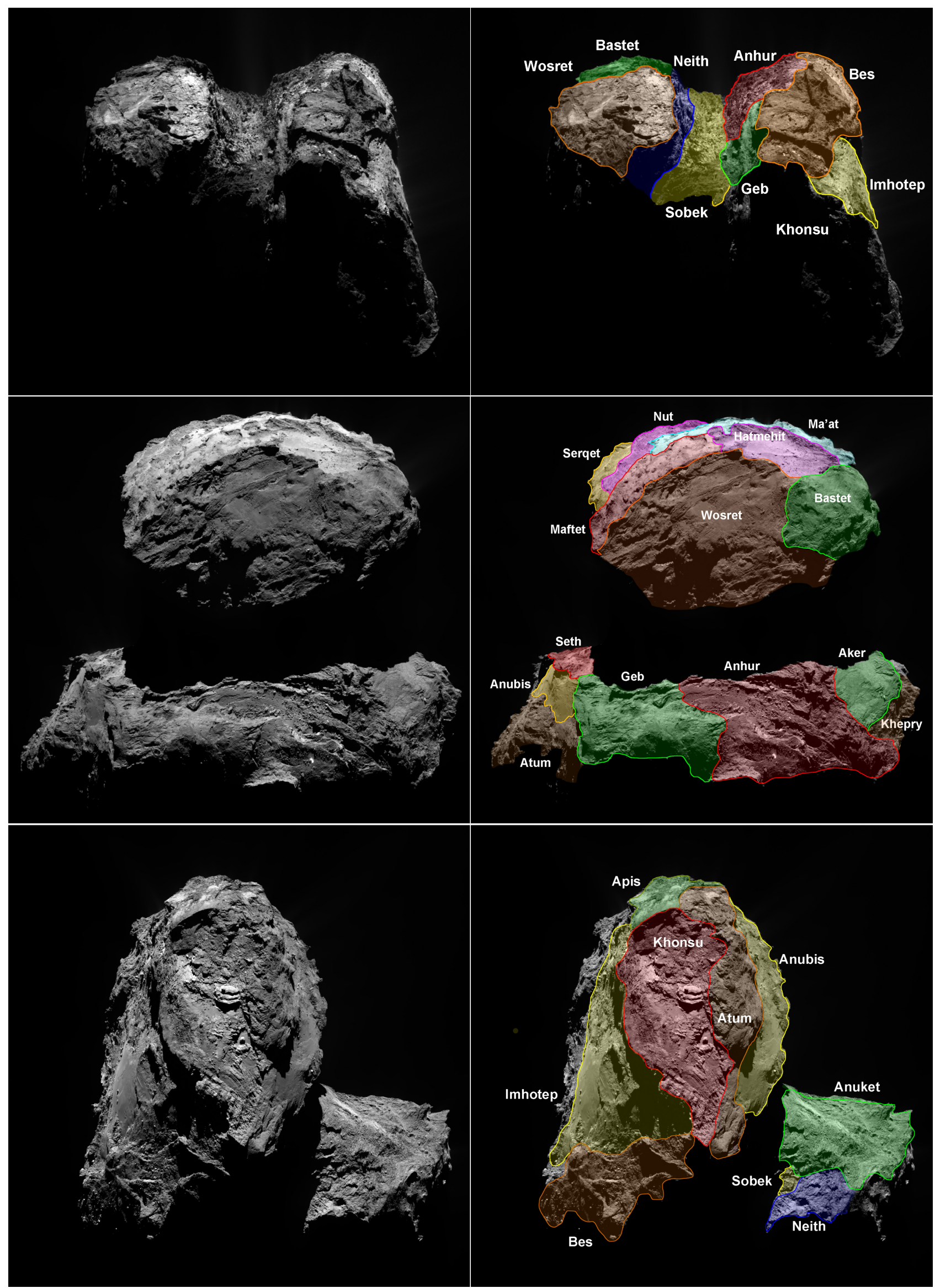

Fig. 1. Different views of the comet's southern hemisphere alongside similar orientations with regional boundaries overlaid. 
Table 1. Distribution of various features of interest that have been discussed in the text.

\begin{tabular}{ccccccc}
\hline \hline Region/feature & Pits & CM & Lay/Terr & Fractures & Smooth deposits & Dust coating \\
\hline Bes (L) & X & X & X & X & X & \\
Khonsu (L) & & X & X & & X & \\
Geb (L) & & & X & X & & \\
Anhur (L) & X & X & X & X & X & \\
Sobek(N) & & X & X & & & \\
Neith(S) & & & X & & & \\
Wosret (S) & X & X & X & $X^{*}$ & X & \\
\hline
\end{tabular}

Notes. The presence of a given structure or feature in a region is marked by an "X". An " $\mathrm{X}$ " " symbol corresponds to either a dominant feature in a region (e.g., dust in Ash) or the highest concentration of a specific feature (e.g., active pits in Seth). Numbers in parenthesis correspond to figures that show a particularly good example of the corresponding feature. The letters "L", "N" and "S" next to the regions' names refer to their position on the comet on the large lobe, neck or head lobe, respectively. The acronyms in the header columns correspond to the following respectively: collapsing material (CM), layering or terraces (Lay/Terr).

\section{Methods and nomenclature}

Since we use methods that were previously employed by El-Maarry et al. (2015a), they will only be summarized here. Unless otherwise noted, all the images presented in this study are taken by the Narrow Angle Camera (NAC) subsystem of OSIRIS, in particular those taken with the so-called orange filter (centered at approx $650 \mathrm{~nm}$ ). The images were taken from a distance range of $\sim 45-125 \mathrm{~km}$ from the comet, which corresponds to approximate spatial resolutions of $\sim 0.8-2.3 \mathrm{~m} / \mathrm{pixel}$, respectively.

The regional boundaries are based on clear morphological and/or topographical boundaries. The used terminology is also similar to El-Maarry et al. (2015a). For instance, "consolidated materials" refers to units that appear rocky and are cohesive enough to display lineaments and fractures (El-Maarry et al. 2015b). "Smooth terrains" refers to units that generally appear to be composed of non-cohesive materials enclosed by rough consolidated units and are particularly thick enough to mask the underlying units. Finally, the term "dust coating" is used to describe materials that show signs of wide-spread mobilization, and appear to be thin enough to reveal outcrops of underlying units.

In addition, we make use of the shape models SHAP4S (Preusker et al. 2015), and SHAP5 (Jorda et al. 2016), which were not available for the earlier study (El-Maarry et al. 2015a), to investigate the topography with higher detail and define structural boundaries with higher confidence. In fact, since the earlier phases of mapping of the southern hemisphere were carried out at large distances from the nucleus, the initial regional definitions were based almost entirely on structural/topographical considerations rather than morphological changes. Therefore, the characterization of the regions was initially carried out by using a 3D shape model of the comet and a computer technique using the software Unity $3 \mathrm{D}^{1}$, which is a visualization platform for the development of graphical applications by means of scripting. Overall the process encompasses two steps. In the first step, the physical boundaries of a region are defined. To do this, we implemented an application to define the boundaries of one region by focusing on the area of interest and clicking with the mouse to start a manual drawing process of a virtual line that defines the region. This line is, in fact, composed of multiple $3 \mathrm{D}$ coordinate points on the surface of the shape model. The exact coordinate points on the surface are obtained by calculating the intersection of a vector (extending from the camera point of view to

\footnotetext{
1 https://unity3d.com
}

the point where a boundary point is chosen) with the polygonal mesh. In the second step, the set of polygons inside the boundary are selected. However, there is neither an easy nor a known trivial method to solve the problem of automatic identification and selection of polygons within an arbitrary 3D shape. Furthermore, the computational cost for a possible automatic technique would be impractical. Therefore, we have implemented a quick manual solution by creating a so-called sphere of adaptive radius over the inner area of a region in order to cover all the polygons inside the boundaries of that region. All the polygons lying inside the volume of the sphere are selected. The radius of the sphere can be reduced to have a better accuracy close to the regional boundaries. This process is repeated until the area of a region is entirely covered. From the set of selected polygons and vertices, the $3 \mathrm{D}$ mesh that represents a region can be generated. As a result, it is possible to display any number of regions in isolation or in any given combination, which aids visualizing and interpreting various structural attributes.

Finally, in accordance with the general theme of the mission and the previous terminology set for the northern hemisphere, the defined regions are named after ancient Egyptian deities. Regions on the small lobe are given female names, whereas regions on the large lobe and the neck region in between are given male ones.

\section{Regional morphology}

In the coming sections, each of the southern regions is described first independently focusing on its overall morphology and important features followed by characterization of the given region's boundary with its neighbors. A summary of various features observed on the surface are provided in Table 1 and the main characteristics of each region are summarized in Table 2. We first start with the regions on the large lobe followed by the neck region and finally the small lobe.

\subsection{Bes}

The Bes region lies on the edge of the large lobe in contact with the Imhotep region, and is essentially the southern polar region of the comet. At first sight, the region appears to be an extension of the northern hemisphere Khepry region, which similarly surrounds the Imhotep smooth terrains. Overall, the region comprises a terraced and fractured surface suggestive of internal layering (Massironi et al. 2015). An interesting feature of note is a sharp scarp in a polygonally-fractured surface, which indicates a 
Table 2. Basic description and morphology of the various regions that are described in the main text.

\begin{tabular}{|c|c|c|c|}
\hline Region & Region type & Description & Notable feature(s) \\
\hline \multicolumn{4}{|c|}{ Large lobe (Body) } \\
\hline Bes & Consolidated & $\begin{array}{l}\text { Rough-looking unit neighboring the Khepry region and enclos- } \\
\text { ing part of Imhotep. Shows evidence for terracing and various } \\
\text { surface textures including pits, fractures, collapsing material, } \\
\text { and patches of smooth material. }\end{array}$ & - An initially bright spot? \\
\hline Khonsu & Consolidated/mixed & $\begin{array}{l}\text { Near-equatorial region, which appears to be depressed in rela- } \\
\text { tion to Atum and Apis regions yet appears to enclose parts of } \\
\text { Imhotep. Includes peculiar outcropping materials, large boul- } \\
\text { ders, patches of smooth deposits and evidence for layering, } \\
\text { which appears to be consistent in orientation with that in neigh- } \\
\text { boring Anubis. }\end{array}$ & - "Pancake" feature \\
\hline Geb & Consolidated & $\begin{array}{l}\text { Part of the main cliff in the southern hemisphere of the large } \\
\text { lobe. Appears to be more consolidated than the neighboring An- } \\
\text { hur region. }\end{array}$ & - Polygonal fracture patterns \\
\hline Anhur & Weakly consolidated & $\begin{array}{l}\text { Second part of the body's southern cliff (along with Geb). Ap- } \\
\text { pears to be weaker than Geb with numerous pits, alcoves and } \\
\text { debris/talus deposits as well as boulder fields. Generally less } \\
\text { steep than Geb. }\end{array}$ & - An initially bright spot? \\
\hline \multicolumn{4}{|c|}{ Neck } \\
\hline Sobek & Consolidated & $\begin{array}{l}\text { Narrow rough region connecting the two lobes of the comet mir- } \\
\text { roring Hapi in the north. Morphologically very different from } \\
\text { the northern neck due to absence of smooth deposits except } \\
\text { for debris and accumulations of dine collapsed material from } \\
\text { surrounding cliffs (especially Anhur). Appears to underlie the } \\
\text { Anuket region and shows a series of terraces moving away from } \\
\text { the boundary with Anuket towards its central part. }\end{array}$ & $\begin{array}{l}\text { - Terraced morphology near the } \\
\text { Anuket boundary }\end{array}$ \\
\hline \multicolumn{4}{|c|}{ Small lobe (Head) } \\
\hline Neith & Consolidated & $\begin{array}{l}\text { The main cliff of the small lobe's southern hemisphere. } \\
\text { Rough, steep and morphologically almost indistinguishable } \\
\text { from Sobek. }\end{array}$ & \\
\hline Wosret & Consolidated & $\begin{array}{l}\text { A flattened unit encompassing most of the face of the small } \\
\text { lobe's southern hemisphere. Shows various and diverse surface } \\
\text { morphology but mainly two distinctive surface textures: heav- } \\
\text { ily fractured areas, and pitted regions. Region is bordered by } \\
\text { Maftet from one side and Bastet on the other, which it appears } \\
\text { to underlie. }\end{array}$ & $\begin{array}{l}\text { - Two bands of layered and } \\
\text { fractured materials roughly par- } \\
\text { allel to the north/south hemi- } \\
\text { spherical boundary }-300 \mathrm{~m} \text { - } \\
\text { long linear fractures near the } \\
\text { Neith cliffs }\end{array}$ \\
\hline
\end{tabular}

Notes. Please refer to the figures in the main text for a visual representation of the extent and boundaries of the different regions.

sharp break-off and collapse of materials (Fig. 2, for information about the images IDs used in each figure, refer to Table A.1). The region also shows diversity in surface textures with numerous scattered boulders of different sizes, shallow pits, debris or talus deposits, and disparate patches of dust covering areas of the region.

As already mentioned, Bes surrounds parts of Imhotep like an elevated rim and is bordered by the cliff regions Anhur and Geb towards the neck and the Khonsu region towards the direction of the Atum and Apis regions in the north by clear topographical boundaries.

\subsection{Khonsu}

The Khonsu region (Fig. 1) is one of the most peculiar regions in the southern hemisphere. It is situated in the southern nearequatorial part. In a sense, it resembles predominantly smooth regions in the northern hemisphere in being slightly depressed with respect to its surroundings. Indeed, it appears to be enclosed bv higher elevated consolidated materials (i.e., the Atum and Apis regions). However, except for a few discontinuous patches of smooth appearance, the region is rough in texture (Fig. 3).
It features a number of irregularly shaped outcrops and a high number of large boulders. The largest boulders (many exceeding $30 \mathrm{~m}$ in diameter) appear to be concentrated around the region's boundaries suggesting a formation through cliff collapse.

A remarkable feature in the Khonsu region is a $200 \mathrm{~m}$-wide composite block of material that appears to be arranged in a stack of 3 plate-shaped features (resembling a "3-stack pancake") rising roughly $220 \mathrm{~m}$ above, and at an inclination $\sim 60$ degrees from the Khonsu plane (Fig. 3). The unique morphology of the feature and the nature by which it outcrops from the surface (see Fig. 4) suggests that it is being gradually exhumed from beneath.

Khonsu is surrounded by the Atum and Apis regions in the north from one side and separated from the Imhotep and Bes regions on the other side by scarps. The Atum ridge rises $\sim 200 \mathrm{~m}$ above the Khonsu plane. Similarly, the Apis region rises above the Khonsu region forming a very sharp scarp (Fig. 4).

\subsection{Geb and Anhur cliffs}

Geb and Anhur represent the cliff regions in the large lobe mirroring Seth on the opposite hemisphere. The two regions display rugged terrain with numerous angular blocks and materials of 

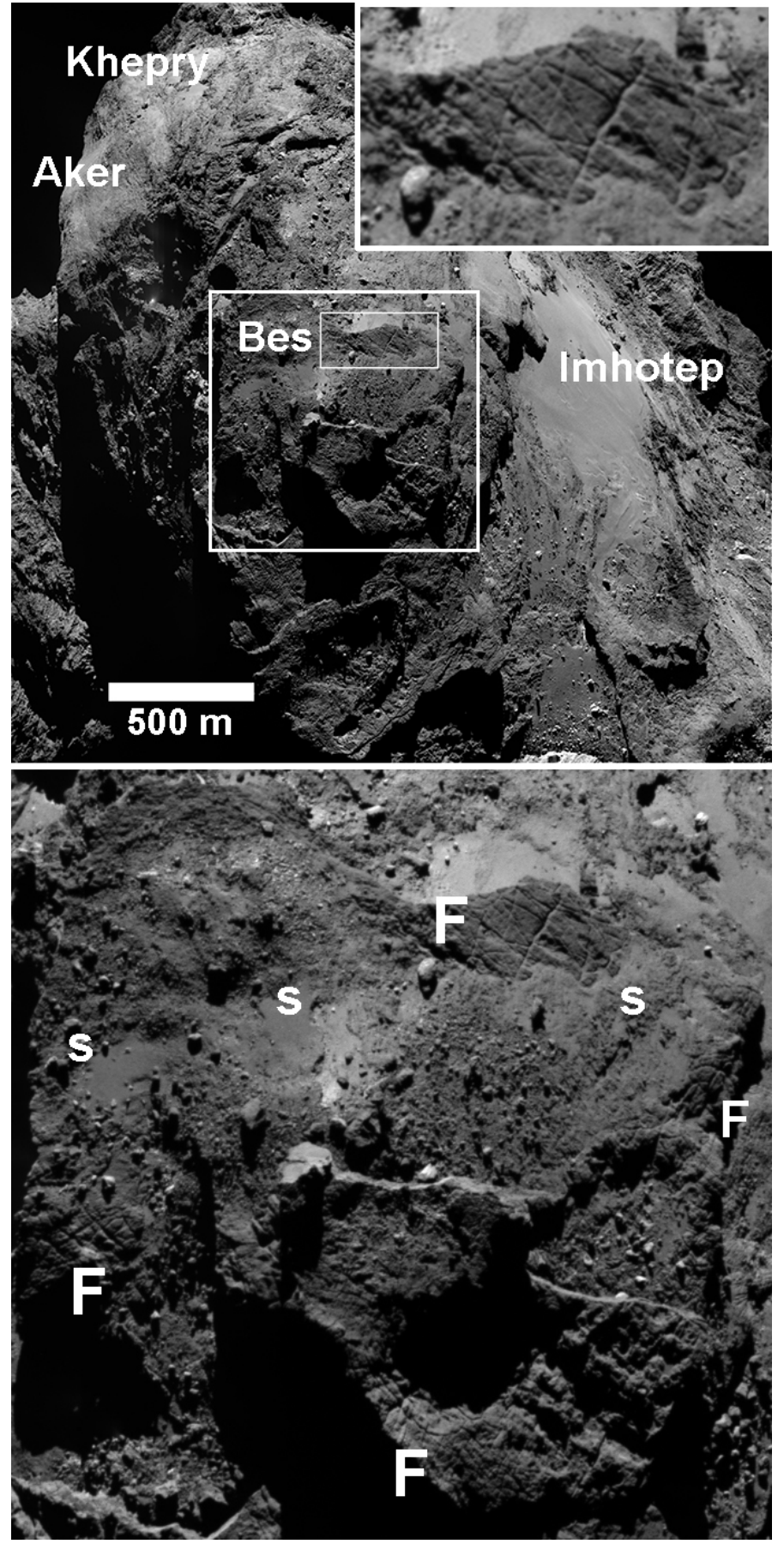

Fig. 2. Upper: NAC image showing the Bes region and surrounding regions. The small box shows the region highlighted in the small inbox, which shows a polygonally-fractured scarp with sharp irregular edge. Larger box shows the position of the zoomed area in the lower panel. Lower: highlighted region in Bes showing the predominantly-fractured surface (marked "F"), which is sporadically covered by irregular smooth coatings (marked "s") of variable size and thickness. For image ID of this figure and others that follow, check Table A.1.

various strengths. Of the two, Anhur appears to be less consolidated as evident from the presence of numerous talus deposits, numerous isolated boulders and boulder fields, and various pits and niches in the walls (Fig. 5). On the other hand, Geb appears to be more consolidated because of its smoother appearance, rarity of boulders and fine debris deposits, and

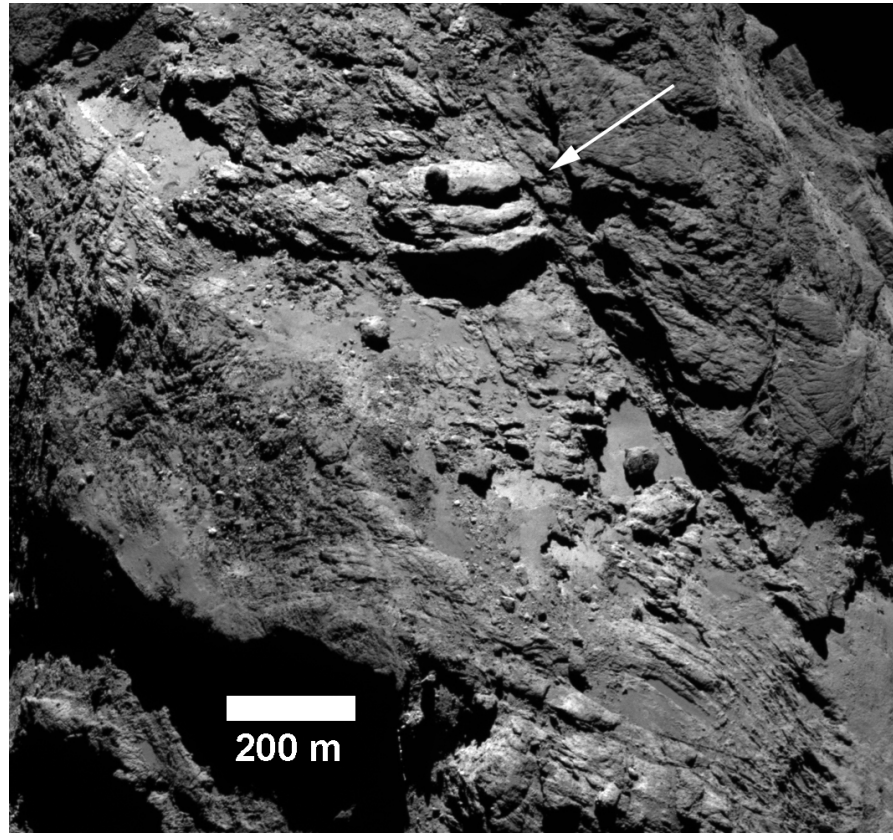

Fig. 3. NAC sub-frame highlighting the Khonsu region including the informally-dubbed "pancake" feature (arrow). The region is rough overall yet includes discontinuous patches of smooth areas and a number of large (10s of meters-wide) boulders.

presence of numerous rectilinear and polygonal fracture patterns (El-Maarry et al. 2015b).

An inspection of digital terrain models of the southern hemisphere (Fig. 6) shows that the boulders in Anhur accumulate at areas where the topographical slope appears to flatten-out, which is consistent with implied weakly-consolidated nature of Anhur compared to the Geb region.

The boundary between both regions is generally gradual and marked by a shift in surface texture from the less consolidated materials of Anhur to the more consolidated and fractured materials of Geb (Fig. 5). In a sense, Anhur could be considered as a sub-region of Geb since the two regions comprise a single structural unit. Nevertheless, the cliff is divided into two regions to highlight this important difference in surface texture. The boundary with Sobek (the neck region) is difficult to discern morphologically and is simply marked by a break in topographical slope. Anhur is also bordered by the northern hemisphere Aker region by a clear morphological and structural transition, and by the Bes region whose boundary marks the head of the cliffs. Geb is similarly bordered by Bes and terminates in another scarp that separates it from the Atum, Anubis and Seth regions in the northern hemisphere (Fig. A.2).

\subsection{Sobek: the southern neck}

The southern hemisphere displays a neck region (Fig. 7) similar to the northern hemisphere (Hapi). However, it is structurally less obvious due to the overall lower relief in the southern hemisphere and morphological similarity of Sobek to surrounding cliffs, especially Neith on the small lobe. No smooth deposits similar to those that cover Hapi in the north are observed. On the other hand, Sobek has a high concentration of boulders, many of them 10s of meters-large, a trait it shares with Hapi, which are likely collapsed materials from the surrounding cliffs of Neith and Anhur. 


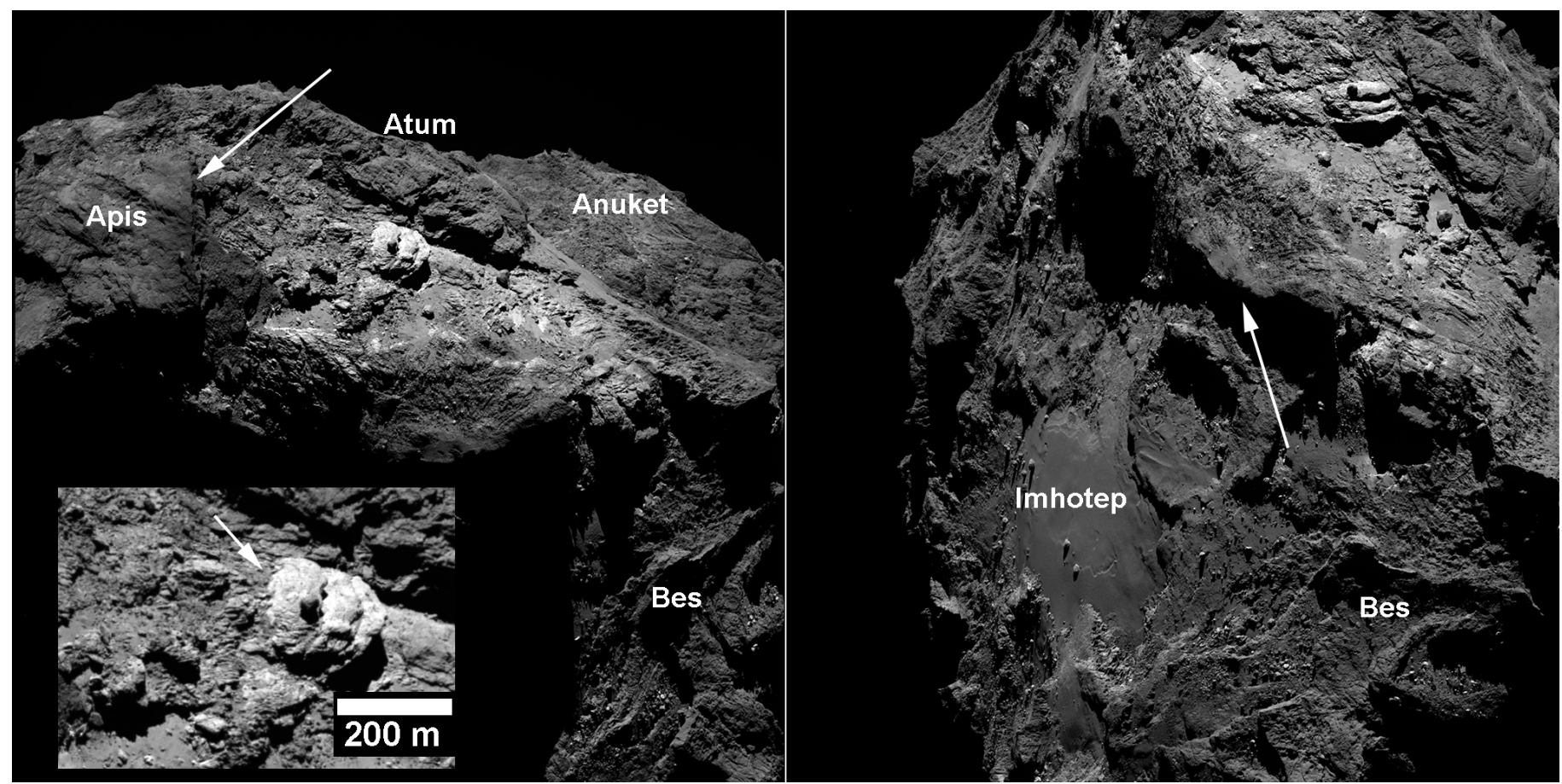

Fig. 4. Left: NAC image showing the Khonsu region highlighting its structural boundaries with Atum, which is separated from Khonsu by an escarpment, and the sharp scarp separating it from Apis (arrow). The inbox shows a close-up of the "pancake" feature highlighting its dip with respect to the surrounding terrain and its contact with the surrounding dark material (arrow), which is indicative of an exhumed nature. Right: another view of the Khonsu region highlighting its boundary with Imhotep (arrow), which is another well-defined scarp.

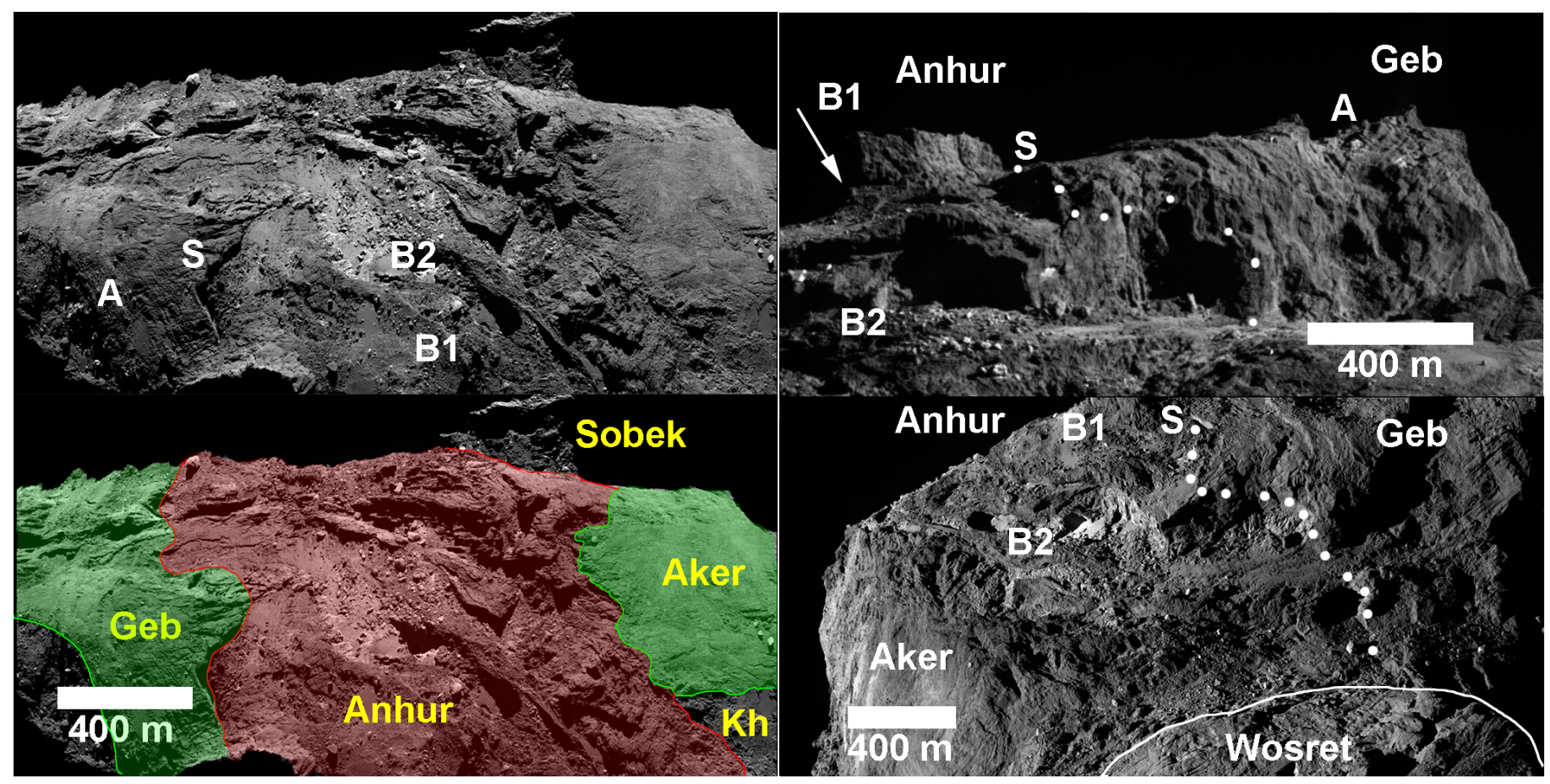

Fig. 5. Left: NAC image showing the main cliff of the large lobe's southern hemisphere from a "top view" along with another version including colored overlays of the defined regions Anhur and Geb and their boundaries. Geb appears to be more consolidated than Anhur as evident from the presence of various fracture patterns (A) and the lack of boulder fields (e.g., B1 and B2 in Anhur). The letter "S" shows the location of a welldefined scarp that separates the two cliff regions. The letters are representative of the same features marked in all panels. Upper right: a "head-on" view of the cliffs highlighting the morphological aspects that have already been mentioned, particularly of Geb. The dots show the location of the boundary marked clearly in the colored overlay of the left panel. Lower right: similar view of the cliffs highlighting the surface texture of Anhur.

Sobek displays morphologically a very rough surface texture that distinguishes it from Anhur and Geb, yet is more difficult to discern from that of Neith, although it is still possible to differentiate between both in some locations where fine debris (probably derived from Anhur) has accumulated covering parts of the Sobek region (see Fig. 8 for instance). As a result, 


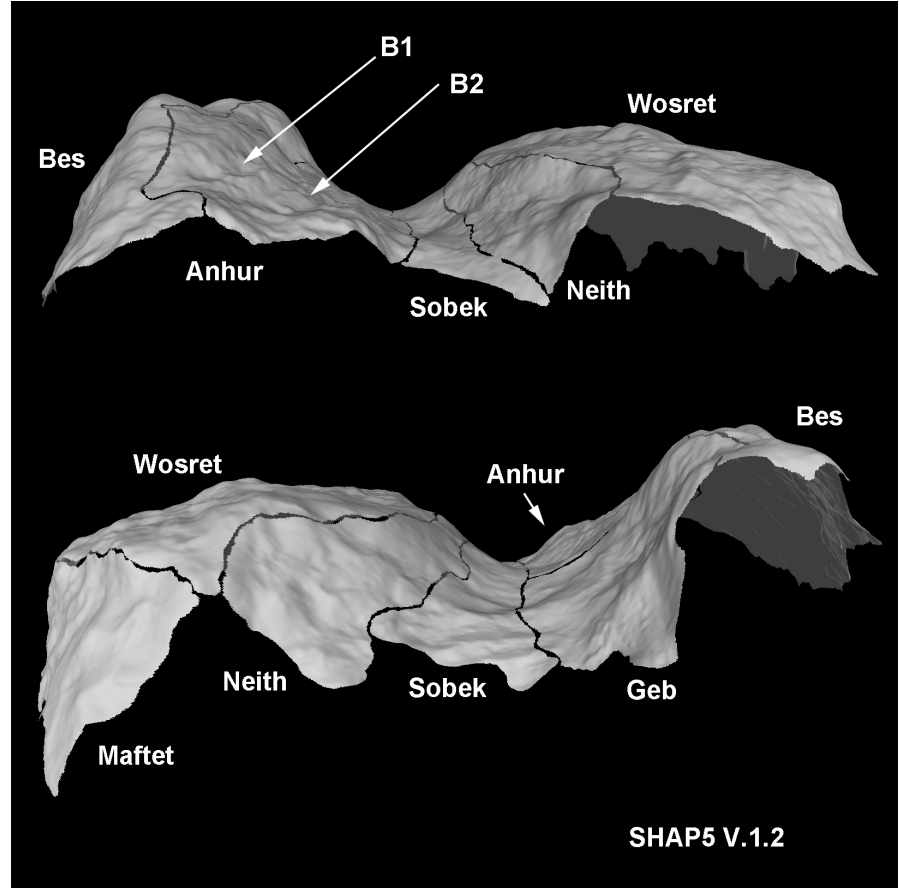

Fig. 6. Two views of digital terrain models for a number of southern hemisphere regions (and Maftet on the small lobe) that highlight the difference in overall slope between the Anhur and Geb sections of the large lobe cliff with respect to the neck region (Sobek, Sect. 3.4) as well as Neith (Sect. 3.5), the main cliff of the small lobe. B1 and B2 are consistent with those of Fig. 5.

the boundary with the Neith cliffs is based solely on topographic considerations and is marked at the point of slope change between the two regions (Fig. 6). On either short sides of Sobek, the region's boundaries with Hapi are clear morphologically due to the transition to smooth surface textures (Fig. 7). Interestingly, the boundaries with Bastet and Anuket, while clear morphologically as well, are both characterized by similar "stratigraphical" traits where the Anuket and Bastet units appear to form gentlysloping scarps and overlie Sobek. In fact, Sobek's boundary with Anuket grades into a series of terraces where Anuket could be considered as the top layer (Fig. 7).

\subsection{Neith: the small lobe's cliff region}

The Neith region represents the main cliff of the small or head lobe. Neith has a lower relief with respect to the neck than that of the large lobe (Anhur and Geb). As discussed earlier, the morphology of Neith is very similar to that of Sobek making it very difficult to discern the two in most NAC images. High resolution images suggest that Neith (and by extension Sobek) is strongly consolidated as evident from the presence of polygonal fractures and lack of evidence for collapsing structures, alcoves or niches. In fact, along with Sobek, the region shows a ridged morphology orientated perpendicular to the comet's long axis (Fig. 8), which may be associated with compression induced by the merging of the lobes (Massironi et al. 2015).

As discussed already, Neith is separated from Wosret and Sobek by topographical boundaries defined at areas of slope change, and from Anuket by a distinctive change in morphology. The boundary with Bastet is characterized by a sharp scarp even more prominent than the one separating Bastet from Sobek (Fig. 7).

\subsection{Wosret}

The Wosret region encompasses roughly the entire southern face of the small lobe. The region appears "flattened-out" with no major changes of relief except for a pitted part situated close to the northern Maftet region. On the distal part of the region a number of quasi-parallel lineaments form two bands that roughly run parallel to the Wosret/Maftet boundary and appear to cross cut the boundary with the Hatmehit depression (Fig. 9). The two bands run almost parallel to each other with a spacing in the range of 115 to $130 \mathrm{~m}$ where the distal band of lineated materials is running adjacent to the northern/southern boundary. Towards the neck region, the regional morphology grades into rougher and more heterogeneous surface textures highlighted by numerous long fractures reaching $\sim 300 \mathrm{~m}$ in length, cross-cut by smaller ones occasionally forming polygonal and rectilinear patterns. High resolution images (less than $1 \mathrm{~m} / \mathrm{pixel}$ ) show that fractures dominate the Wosret landscape with various settings and morphologies (Fig. 10).

Wosret borders the Neith cliffs towards the neck region, marked by an abrupt change in slope, and Maftet and Hatmehit on the distal part of the lobe, marked by clear topographical and morphological boundaries. Wosret also borders the Bastet region on one side and the Anuket region on the other. The boundary with Bastet is structural as a scarp separates the two regions and Bastet appears to overlie Wosret (Figs. 7 and A.4). Conversely, the small boundary with Anuket is morphologically gradual as the surface textures grades into the so-called melted wax texture of Anuket (El-Maarry et al. 2015a).

\subsection{Addendum to northern hemisphere regions}

El-Maarry et al. (2015a) asserted that some of the northern hemisphere regions, mainly equatorial ones, may need updating following the illumination of the southern hemisphere. In most cases, these regions where either shown to be in no need of further modifications, such as Apis and Maftet, because of their exceptionally sharp boundaries (check Figs. 4 and 9, respectively), or because they displayed minor additional terrains that appear to be morphologically indistinguishable from their overall morphology, such as Aker. Therefore, we are only highlighting here the most prominent additions in two regions: Imhotep and Bastet.

\subsubsection{Imhotep}

Figure A.3 shows the area that has been added to Imhotep. The newly added terrain shares the same setting with Imhotep in being enclosed by the rougher-looking regions of Bes and Khonsu. In addition, it displays similar surface morphology characterized by smooth-looking plain deposits covering rougher-textured materials (Auger et al. 2015). The area also displays the remaining extent of a large circular feature that was displayed partly before equinox, and rough-textured terrains that show less coverage by smooth deposits and numerous boulders of different sizes, particularly concentrated close to the region's boundary.

A more detailed study of these new terrains in addition to morphological changes to Imhotep, first reported in Groussin et al. (2015b), is to be presented by Auger et al. (in prep.). 

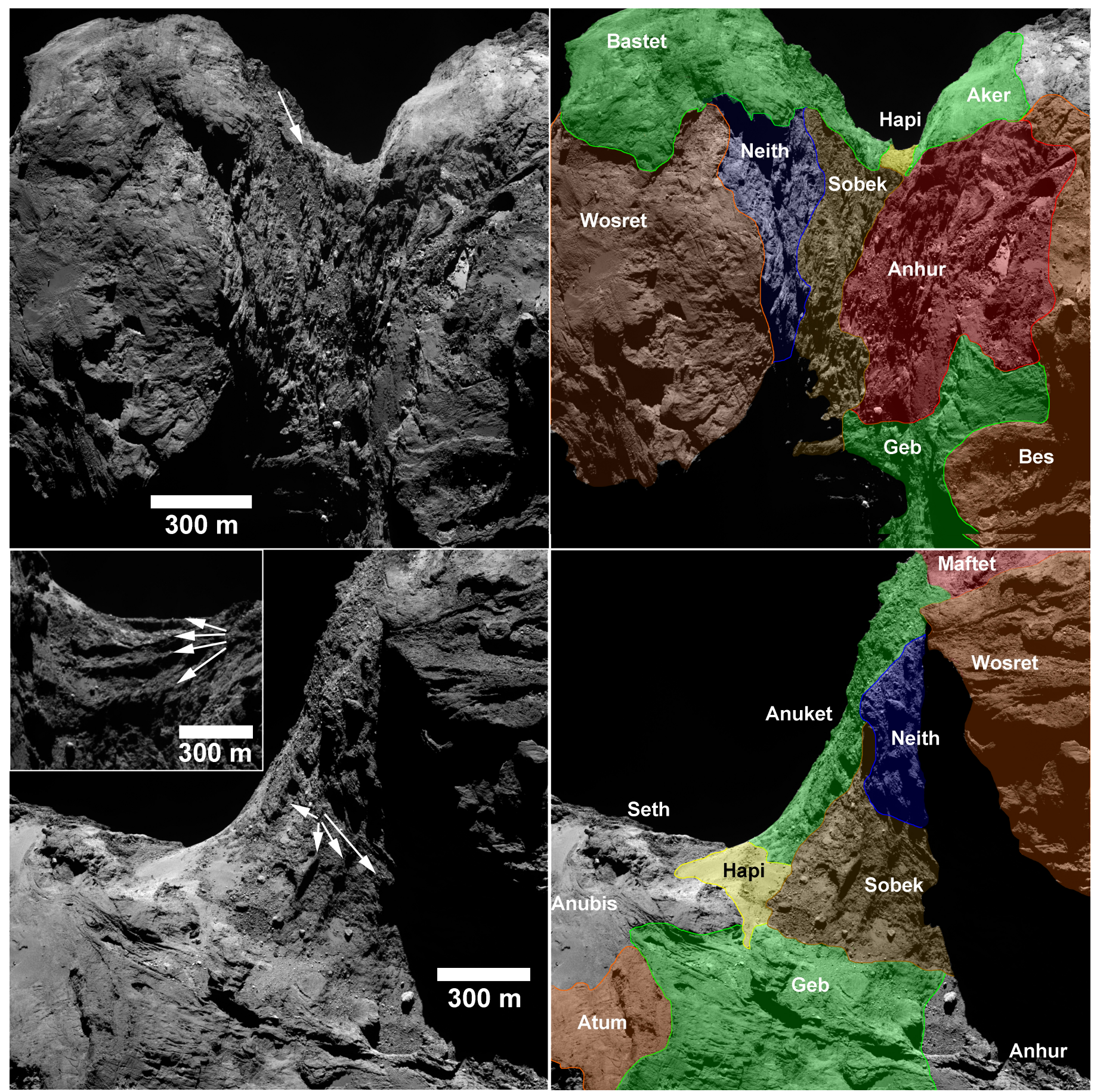

Fig. 7. Top: NAC image showing a "view from the top" of the southern hemisphere including a significant portion of the neck region Sobek along with a similar view including a colored overlay of the regions and their boundaries. Note the difficulty in differentiating Sobek from Neith on account of morphology. The arrow highlights the boundary with Bastet, which appears to overlie the neck region. Bottom: another view of the southern hemisphere with a similar color overlay highlighting the boundaries of Sobek with neighboring regions on the side opposite to that shown in the top panel. Note the arrows that show evidence for terraces starting from the contact with Anuket, which is better shown in the view taken at different geometry and illumination conditions (inbox). As the image in the box was taken from a longer distance (i.e., lower resolution), it has been resampled using a bicubic interpolation in order to show the terraces more clearly.

\subsubsection{Bastet}

Figure A. 4 shows the areas that have been added to the Bastet region. The new areas encompass parts of Bastet that appear to be morphologically similar to its northern hemisphere part yet are darker in tone, possibly caused by lack of lighter-toned coatings, as well as being more fractured. The new areas of Bastet form the boundary with Wosret and are clearly overlying the fractured plains of the southern region. The Bastet/Wosret boundary appears to be a source of debris and boulders in the vicinity.

\section{Discussion}

\subsection{Comet 67P's hemispherical dichotomy}

As has been demonstrated in the previous sections, the general morphology of the southern hemisphere closely resembles 

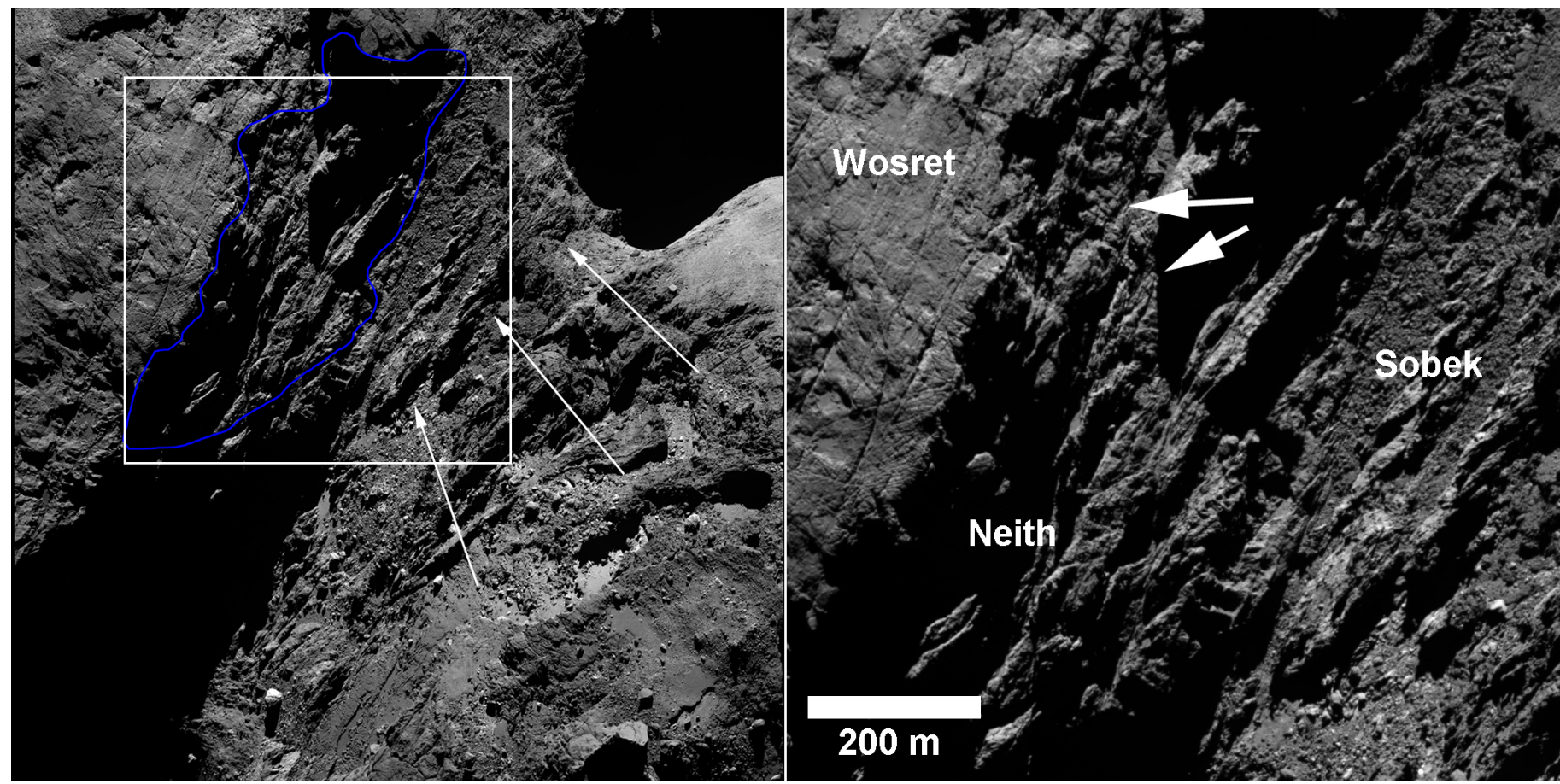

Fig. 8. Left: NAC image of the southern hemisphere showing parts of Neith and neighboring regions. A blue polygon outlines the approximate boundaries of the region, and the inbox shows the location highlighted in full resolution in the right panel. Notice how the debris derived from Anhur appears to cover areas of Sobek, which partially aids in discerning Sobek from Neith (arrows). Right: a full resolution cut-out from the image shown in the left panel highlighting the morphology of Neith and its similarities with Sobek and Wosret. Arrows point to locations of polygonal fractures similar in morphology and size-scale to some of those observed in Wosret.

the northern hemisphere consolidated regions (El-Maarry et al. 2015a). Nevertheless, the southern hemisphere shows a clear dichotomy with the north most notably because of the lack of wide-spread dust coatings and smooth terrains. In addition, large depressions are markedly absent in the southern hemisphere, despite the presence of numerous irregular pits, niches, and alcoves, especially in the cliffs. Together, the lack of these features decreases the textural diversity in the south compared to the northern hemisphere. In the absence of such features, the regions' dominating features are fractured and patterned rough terrains, and less consolidated areas displaying talus deposits and boulder fields, particularly in the Anhur region.

Overall, the southern hemisphere shows significantly less topographical variation and looks flattened-out in comparison to the northern hemisphere, which may be a direct result of higher insolation input to the southern hemisphere, which it receives during its short, but intensive, summer that closely coincides with perihelion passage. Keller et al. (2015) calculated that the southern hemisphere should experience up to a factor of 3 higher erosion rates, which could offer an explanation for the overall lower relief in the south. However, a more challenging observation to explain is the apparent absence of large deposits of smooth materials and dust coating in the southern hemisphere. One possibility would be that the higher activity in the southern hemisphere is releasing a larger proportion of particles at speeds exceeding the escape velocity, thereby preventing them from settling back on the surfacein contrast to the situation envisioned for the northern hemisphere (Thomas et al. 2015a).

\subsection{Latitudinal trends}

Figure 11 shows a regional map of all regions defined on the surface of comet $67 \mathrm{P}$ in a cylindrical projection, which was prepared, in part, to investigate possible latitudinal trends. In this configuration, we can see that large-scale depressions (Aten, Hatmehit, and Nut) fall roughly in equatorial to mid-latitude regions. As already mentioned, dust-covered (Ash and Ma'at), and smooth terrains-dominated regions (Hapi, Imhotep, and Anubis) are located only in the northern hemisphere. Apart from these distributions, no discernible trends can be observed that would indicate latitudinal control on morphology or geologic setting. It is interesting to note that whereas the northern neck (Hapi) is essentially the northern polar region, due to the inclination of the comet's axis of rotation, it is the Bes region, not the southern neck (Sobek), which lies in the southern polar position.

\subsection{Khonsu vs. other large-scale depressions}

As already presented in Sect. 3.2, Khonsu is a unique and intriguing region on comet $67 \mathrm{P}$. In a sense, Khonsu could be considered as a "depression" in relation to the neighboring Atum and Apis regions, which lie relatively on the same face of the comet as Khonsu (as opposed to the neighboring Imhotep for instance). Furthermore, the sharp contact with Apis is similar to the boundaries exhibited by the Aten depression in the northern hemisphere, which was interpreted to be a possible evidence for outburst activity removing large chunks of materials (El-Maarry et al. 2015a). Indeed, in favorable viewing angles (Fig. A.5), Khonsu could be considered as a location where the internal structure of the comet's large lobe is exposed due to removal of surface materials coupled with lack of smooth materials or dust coatings that have obscured the interiors of similar depressions in the northern hemisphere. If that is the case, then the unique "pancake" feature in this region (Figs. 3, 4) may be of primordial origin incorporated during the accretion process. This hypothesis is consistent with its regional setting as being 

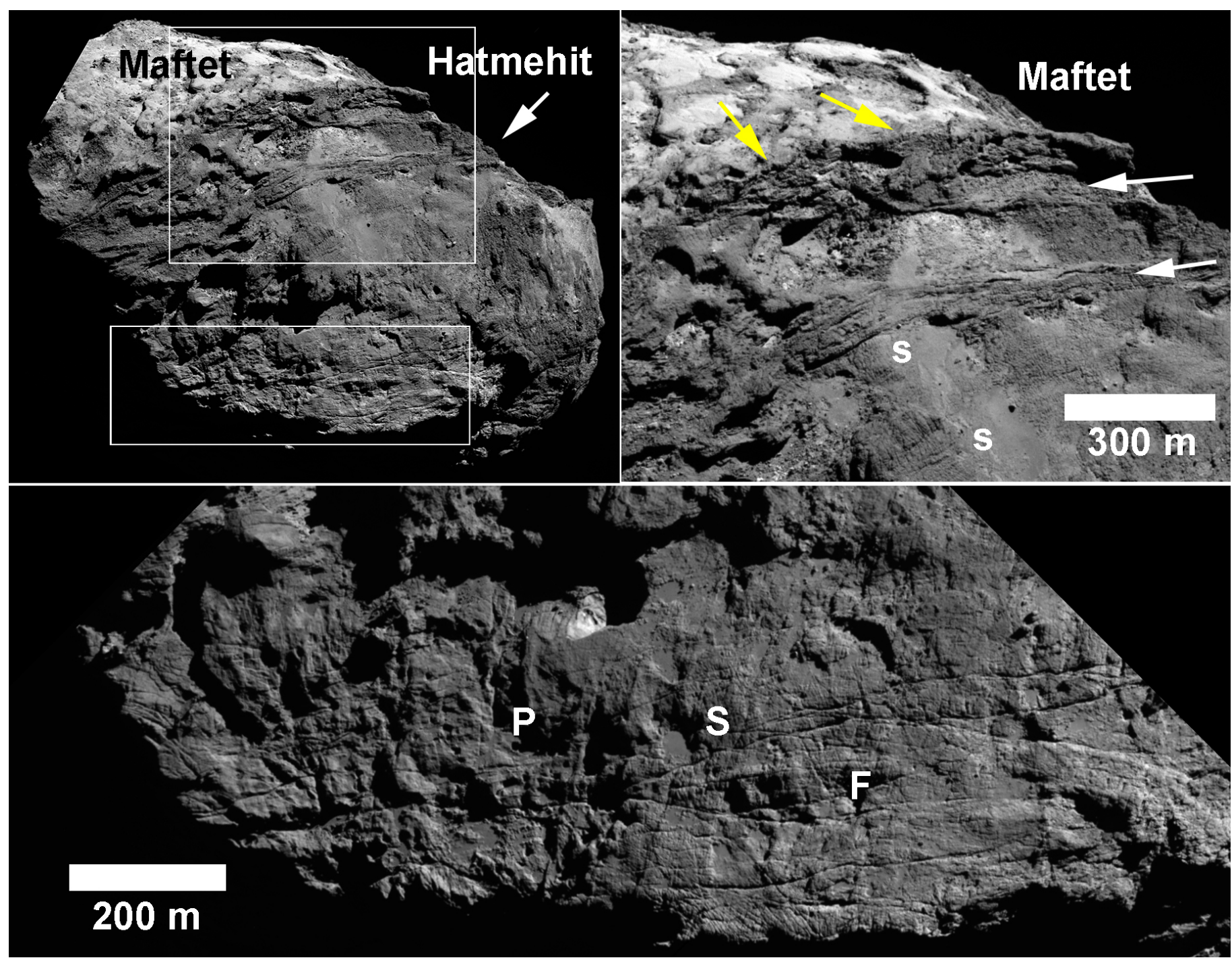

Fig. 9. Top left: NAC image showing the Wosret regions. The boxes show the location highlighted in the other panels. Top right: a section of the same NAC image like the top left panel at full resolution highlighting the banded morphology. A number of quasi-parallel lineaments form roughly two bands (white arrows) that run almost parallel to the Wosret/Maftet boundary (yellow arrows). Note the patches of smooth material (S). Bottom: another NAC image taken at a closer distance showing the lower part of the Wosret region close to the boundary with Neith. Two main surface textures can be resolved: a pitted terrain $(\mathrm{P})$, which further extends upwards towards the Maftet boundary, and an apparently more consolidated and fractured terrain $(\mathrm{F})$ displaying long quasi-linear fractures reaching $300 \mathrm{~m}$ in length cross-cut by shorter fractures and forming polygonal patterns in some areas. Some pits appear to have been filled by smooth materials (S). The uneven edges of the image are due to to rotation of the image for better viewing.

exhumed from the comet's subsurface (see Fig. 4). However, it does not offer a satisfactory explanation for its unique shape.

We note that during this comet's closely monitored perihelion passage, and despite the occurrence of numerous outbursts of activity, there has been no large-scale morphological changes that would confirm or strengthen the hypothesis of Khonsu's formation through a massive outburst. Therefore, while the hypothesis fits well with the current surface morphology, its mechanism of formation is yet to be observed.

\subsection{Homogeneity vs. heterogeneity of comet 67P: new considerations}

With the newly acquired information about the surface morphology of the southern hemisphere, it is possible to draw a preliminary global picture of the nucleus as a whole. So, we would like to highlight here a few observations that could be relevant in understanding the degree of homogeneity/heterogeneity of comet $67 \mathrm{P}$ as a whole as well as possible differences between the two lobes themselves. However, it is important to clarify in the process the nature, and scale, of the inferred homo/heterogeneity, especially in comparison to previous studies that have attempted to address this admittedly challenging topic.

Generally, current relevant studies fall into two broad categories: those that investigate 1) bulk properties as directly inferred from certain instruments such as the Radio Science Investigation (RSI) experiment (Pätzold et al. 2016), and the Comet Nucleus Sounding Experiment by Radio wave Transmission (CONSERT) instrument (Kofman et al. 2015), and those that study 2) surface properties, which may be used to infer surface heterogeneity, and possibly bulk properties. In the first category, the homogeneity/heterogeneity in question is mainly concerned with density. For instance, Pätzold et al. (2016) state that "the 


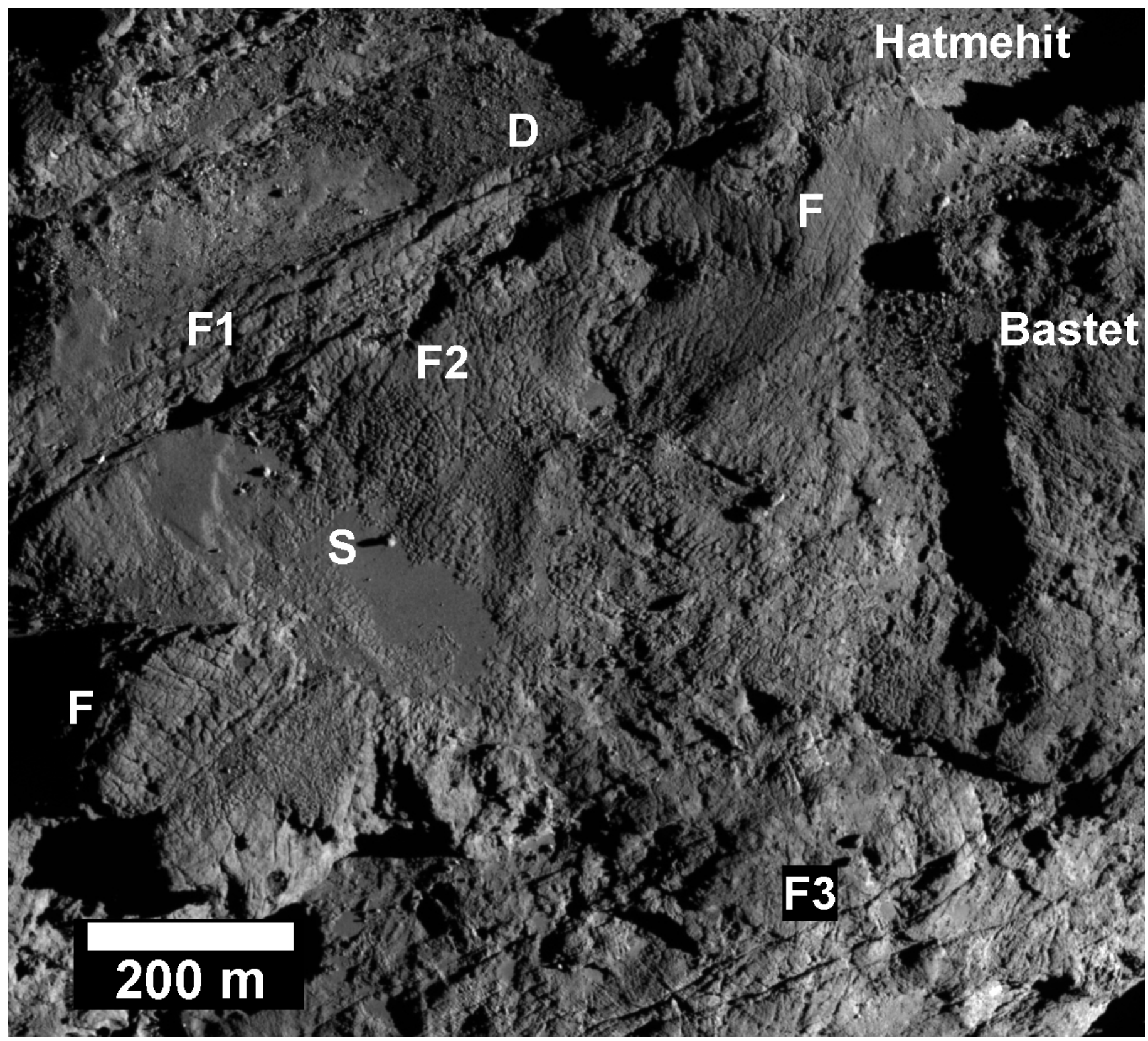

Fig. 10. NAC image highlighting the fractured terrains of the Wosret region. Fractures are ubiquitous in the area (all areas marked with an "F") and appear to dominate the surface texture. For instance, fractures are visible in association with the linear bands (F1), as well plain surfaces, forming polygonal patterns (F2). Both fractures are different from the long linear fractures (F3) visible close to the Neith cliffs. Note that smooth patches of material appear to overly the patterned grounds (S) in addition to coarser-grained material (D) that appears to be a result of collapsing material.

interior of the nucleus is homogeneous and constant in density on a global scale without large voids", and while they do not explicitly mention the size scale of the mentioned voids, they cite similar findings from Kofman et al. (2015) who conclude that the "head", or the small lobe, is "homogeneous down to the scale of tens of meters". Alternatively, Gutiérrez et al. (2016) conclude that a homogeneous body cannot adequately explain comet 67's precession and complex rotation, and suggest instead that the computed moments of inertia are more consistent with a heterogeneous body. This is also consistent with a new study of OSIRIS-derived shape models by Jorda et al. (2016) who conclude that the coordinates of the center of gravity are not compatible with a uniform nucleus density.

As an example of the second category, Massironi et al. (2015) analyzed stratification/layering orientations in both lobes and concluded that the global layering pattern is different in both lobes, which suggests that they formed independently and later merged through low velocity collisions. This merging scenario is corroborated by two additional studies by Rickman et al. (2015), and Davidsson et al. (2016). However, while Rickman et al. (2015) suggest that comet 67P may be a collisional rubble pile, Davidsson et al. (2016) alternatively prefer a primordial rubble pile origin. Despite their different conclusions, both these studies envisage scenarios where both lobes may have variable densities or properties. In another study, Vincent et al. (2015) reported on the distribution of "active pits" in the northern hemisphere and suggested that "the size and spatial distribution of pits imply that large heterogeneities exist in the physical, structural or compositional properties of the first few hundred metres below the current nucleus surface" (Vincent et al. 2015). 


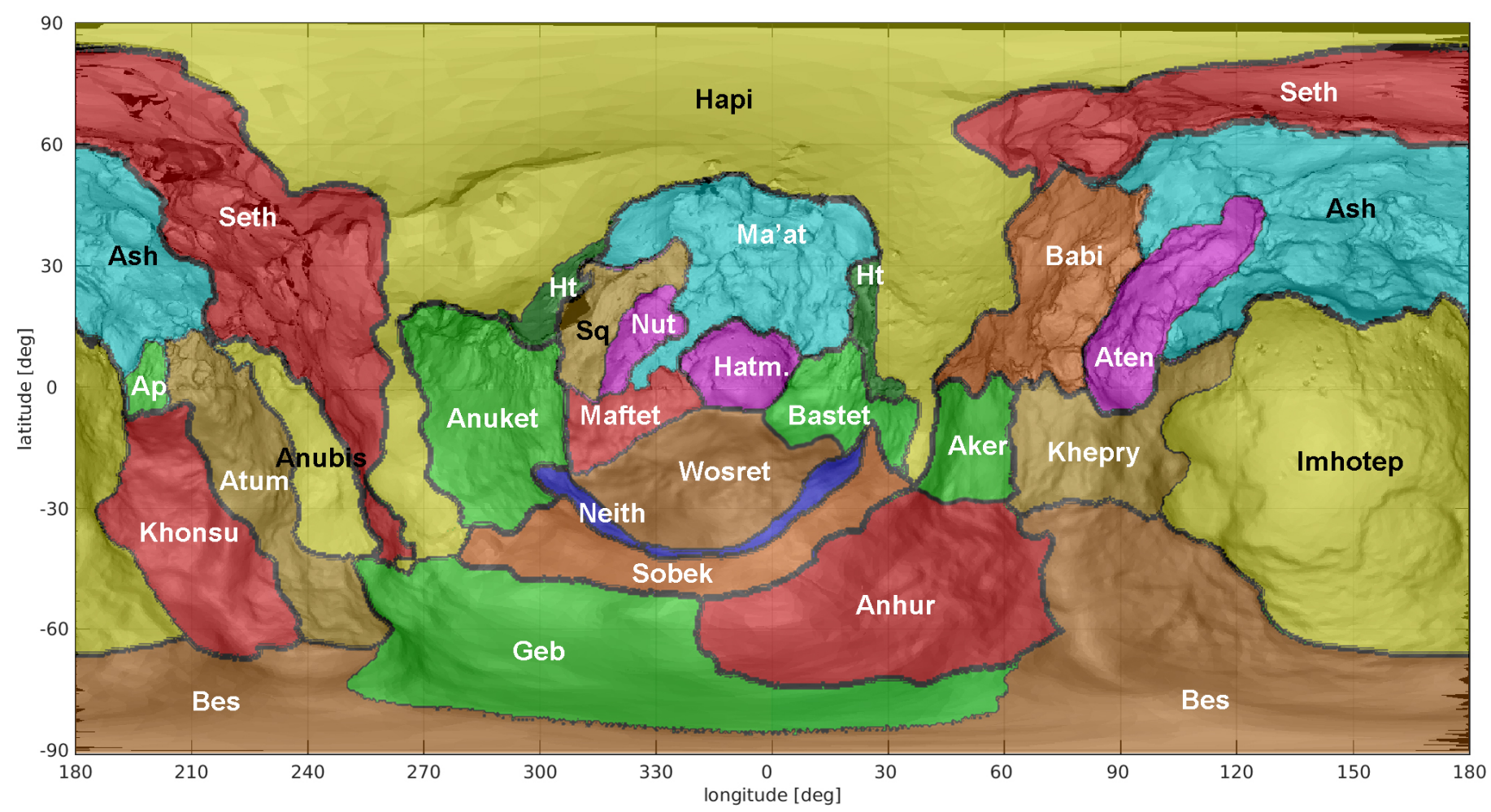

Fig. 11. Map of comet 67P in a cylindrical projection with all defined regions as colored overlays. Acronyms are as follows: Ap: Apis, Ht: Hathor, Sq: Serqet, and Hatm.: Hatmehit. For more information regarding the northern hemisphere regions, refer to El-Maarry et al. (2015a).

Our findings in this study broadly fall in the second category. We use the morphological attributes displayed by the numerous regions on comet $67 \mathrm{P}$ to gain an insights into two main questions: 1) do the comet's lobes display different physical properties?; and 2) does the comet display a heterogeneous surface in the sense that different regions have unique morphologies and/or react to erosional processes differently, which would imply differences in composition or degree of consolidation?

Regarding the comet's lobes, there are a number of interesting observations:

- The cliffs of the small lobe appear to have higher strengths as evident from their higher steepness, and the lack of associated debris deposits compared to the less steep and apparently less consolidated Seth and Anhur regions on the body lobe. In addition, large-scale (10s to hundreds of meters in size) fracture patterns are predominantly observed in the small lobe. Notable examples are the long isolated fracture cutting through the Anuket and neck region, and the Hathor vertical fractures in the northern hemisphere (El-Maarry et al. 2015a), and the $300 \mathrm{~m}$-long fractures in Wosret in the south. The presence of such large-scale fractures is an indication that the materials have high enough strength to support such features without collapsing.

- While most of the comet's regions fall broadly into a number of common categories, some regions show unique morphologies that have no analogues on the opposite lobe such as Anuket, Hathor, and Wosret on the small lobe, as well as Aker, Khonsu and Imhotep on the large lobe.

Taken together, this suggests that the two lobes of comet 67P show regions of variable strength and unique surface textures, which is indicative of different physical and mechanical properties and possibly composition. In this respect, it is important to place these conclusions in the context of results from other
Rosetta instruments. For instance, the Visible, Infrared and Thermal Imaging Spectrometer (VIRTIS) early results (Capaccioni et al. 2015) indicated an organics-rich surface for comet 67P but suggested that the surface of both lobes was homogeneous in composition. However, these results were reported for the early phases of the mission (Aug. to Sep., 2014) when the spatial resolution was between 15 and $30 \mathrm{~m} /$ pixel, and the comet was still at 3.6 to $3.3 \mathrm{AU}$ away from the Sun. Follow-up studies at higher spatial resolutions of 2.5 to $25 \mathrm{~m} / \mathrm{pixel}$ (Filacchione et al. 2016) that carried out direct comparisons between the 19 regions in the northern hemisphere conclude that the northern regions fall broadly into three spectral classes, and that interestingly, the consolidated terrains are spread over all three classes, which would be consistent with possible compositional/mechanical differences. However, Filacchione et al.'s work does not show any direct evidence for compositional or spectral differences between both lobes.

It is interesting to note that variations of organic content, which Filacchione et al. (2016) indeed report could lead to a dual change in both composition and mechanical strength by varying the degree of sintering. Such a process has been demonstrated in experiments aimed at modeling the evolution of cometary analogues through sublimation in the presence of organics (e.g., Grun et al. 1991; Kömle et al. 1996; Kochan et al. 1998). In addition, sintering has been suggested as a mechanism for formation of the hard materials encountered by the Philae lander in its final landing site, as measured by the Multipurpose Sensors for Surface and Sub-Surface Science (MUPUS, Spohn et al. 2015), to be more than $2 \mathrm{MPa}$ in compressive strength.

Finally, if we were to look further, such surface heterogeneities are not confined to differences among certain regions, but may also extend to local differences (e.g., within single regions). Many regions on both lobes show a degree of homogeneity in their surface morphology. Prominent examples include 
Seth, Hathor, Anuket, Atum, Aker, and the major depressions. On the other hand, other regions include unique features in what may otherwise be considered a homogeneous terrain. Examples include the clusters of circular pits in Imhotep, and the "pancake" feature in Khonsu. However, there are certain regions that show sudden changes in morphology and physical characteristics. In this study, two such cases are evident. The first one is the abrupt transition in apparent material strength between the Anhur and Geb regions. While both areas represent two regions on account of their morphology, they are in fact one structural unit as together they represent the main cliff in the large lobe. There is no simple explanation for the abrupt change in their characteristics given their similar location on the body lobe, orientation with respect to insolation patterns, and latitude. The second case is the Wosret region where there is another abrupt change in surface texture from fractured to pitted terrains. While it is possible that the fractured terrains might be overlying the pitted terrains, this would nonetheless indicate abrupt vertical changes, which are similarly difficult to explain using the homogeneous density paradigm for comet 67P's bulk properties.

\section{Summary and conclusions}

We have defined 7 regions in the southern hemisphere of comet $67 \mathrm{P}$ bringing the total of defined regions to 26 on the surface of the nucleus. The southern part of the large lobe encompasses the Bes region, which surrounds the Imhotep smooth terrains and physically resembles the Khepry region in the north. Bes and Anhur represent the main cliff rising above the southern neck and are characterized by notable differences in surface texture where Geb shows stronger degree of consolidation and higher slopes as opposed to Anhur, which features a gentler slope, scattered boulder fields, debris deposits, and numerous niches and alcoves. Also on the large lobe is a peculiar equatorial region named Khonsu, which is morphologically diverse and notable for the presence of a unique feature that appears to have been exhumed from the comet's interior.

The large lobe is connected to the small lobe by a narrow neck region (Sobek), which is very rough in morphology, unlike its northern counterpart, and is almost indistinguishable from the cliff region of the small lobe (Neith). The morphology of these two regions is consistent with highly consolidated materials possibly due to compressional conditions induced by merging of the lobes. The entire southern face of the small lobe is defined as the Wosret region, which displays two types of terrain: pitted terrains showing a high density of depressions particularly towards the boundary with the northern Maftet region, and heavily fractured terrain that displays unique banded features.

The southern hemisphere shows a remarkable dichotomy with its northern counterpart mainly due to the absence of widescale smooth terrains, dust coatings and large unambiguous depressions. As a result, the southern hemisphere closely resembles previously identified consolidated regions of variable mechanical strength. Our assessment of the overall morphology of comet $67 \mathrm{P}$ suggests that the comet's two lobes may have different physical/mechanical characteristics. While other instruments such as VIRTIS do not offer spectral evidence for this inter-lobe heterogeneity, it does indicate a degree of heterogeneity among the different regions on the comet including variations among the consolidated regions themselves. Additional sharp morphological changes within the regions themselves further indicate physical/mechanical, and possibly compositional, heterogeneity of the surface. Such differences, while indirectly inferred here, may yet be directly verified by other Rosetta instruments. This would especially be possible during the final phases of the mission as the spacecraft comes even closer to the comet's surface and, in return, sufficiently improve the spatial resolution of onboard instruments to detect smaller-scale variations.

Acknowledgements. OSIRIS was built by a consortium of the Max-PlanckInstitut für Sonnensystemforschung, in Göttingen, Germany, CISAS-University of Padova, Italy, the Laboratoire d'Astrophysique de Marseille, France, the Instituto de Astrofísica de Andalucia, CSIC, Granada, Spain, the Research and Scientific Support Department of the European Space Agency, Noordwijk, The Netherlands, the Instituto Nacional de Técnica Aeroespacial, Madrid, Spain, the Universidad Politećhnica de Madrid, Spain, the Department of Physics and Astronomy of Uppsala University, Sweden, and the Institut für Datentechnik und Kommunikationsnetze der Technischen Universität Braunschweig, Germany. The support of the national funding agencies of Germany (DLR), France (CNES), Italy (ASI), Spain (MEC), Sweden (SNSB), and the ESA Technical Directorate is gratefully acknowledged. We thank the ESA teams at ESAC, ESOC and ESTEC for their work in support of the Rosetta mission.

\section{References}

Auger, A.-T., Groussin, O., Jorda, L., et al. 2015, A\&A, 583, A35

Capaccioni, F., Coradini, A., Filacchione, G., et al. 2015, Science, 347, 628 Davidsson,B., Sierks, H., Güttler, C., et al. 2016, A\&A, 592, A63

El-Maarry, M. R., Thomas, N., Giacomini, L., et al. 2015a, A\&A, 583, A26

El-Maarry, M. R., Thomas, N., Gracia-Berná, A., et al. 2015b, Geophys. Res. Lett., 42, 5170

Filacchione, G., Capaccioni, F., Ciarniello, M., et al. 2016, Icarus, 274, 334 Groussin, O., Jorda, L., Auger, A.-T., et al. 2015a, A\&A, 583, A32 Groussin, O., Sierks, H., Barbieri, C., et al. 2015b, A\&A, 583, A36 Grun, E., Kochan, H., \& Seidensticker, K. J. 1991, Geophys. Res. Lett., 18, 245 Gutiérrez, P. J., Jorda, L., Gaskell, R. W., et al. 2016, A\&A, 590, A46 Jorda, L., Gaskell, R., Capanna, C., et al. 2016, Icarus, 277, 257 Keller, H. U., Barbieri, C., Lamy, P., et al. 2007, Space Sci. Rev., 128, 433 Keller, H. U., Mottola, S., Davidsson, B., et al. 2015, A\&A, 583, A34 Kochan, H., Huebner, W. F., \& Sears, D. W. G. 1998, in Laboratory astrophysics and space research, eds. P. Ehrenfreund, C. Krafft, H. Kochan, \& V. Pirronello, Astrophys. Space Sci. Libr., 236, 623

Kofman, W., Herique, A., Barbin, Y., et al. 2015, Science, 349

Kömle, N. I., Kargl, G., Thiel, K., \& Seiferlin, K. 1996, Planet. Space Sci., 44, 675

Massironi, M., Simioni, E., Marzari, F., et al. 2015, Nature, 526, 402 Pajola, M., Vincent, J.-B., Güttler, C., et al. 2015, A\&A, 583, A37 Pätzold, M., Andert, T., Hahn, M., et al. 2016, Nature, 530, 63 Pommerol, A., Thomas, N., El-Maarry, M. R., et al. 2015, A\&A, 583, A25 Preusker, F., Scholten, F., Matz, K.-D., et al. 2015, A\&A, 583, A33 Rickman, H., Marchi, S., A'Hearn, M. F., et al. 2015, A\&A, 583, A44 Sierks, H., Barbieri, C., Lamy, P. L., et al. 2015, Science, 347, 1044 Spohn, T., Knollenberg, J., Ball, A. J., et al. 2015, Science, 349 Thomas, N., Davidsson, B., El-Maarry, M. R., et al. 2015a, A\&A, 583, A17 Thomas, N., Sierks, H., Barbieri, C., et al. 2015b, Science, 347, 440 Vincent, J.-B., Bodewits, D., Besse, S., et al. 2015, Nature, 523, 63

1 Physikalisches Institut, Sidlerstr. 5, University of Bern, 3012 Bern, Switzerland e-mail: mohammed.elmaarry@space.unibe.ch

2 Centro di Ateneo di Studi ed Attivitá Spaziali, "Giuseppe Colombo" (CISAS), University of Padova, 35131 Padova, Italy

3 Department of Earth Sciences, National Central University, 32054 Chung-Li, Taiwan

${ }^{4}$ Dipartimento di Geoscienze, University of Padova, via G. Gradenigo 6, 35131 Padova, Italy

5 Jet Propulsion Laboratory, M/S 183-301, 4800 Oak Grove Drive, Pasadena, CA 91109, USA

${ }^{6}$ Solar System Exploration Research Virtual Institute, Southwest Research Institute, 1050 Walnut St., Suite 300, Boulder, CO 80302, USA

7 Institute for Geophysics and Extraterrestrial Physics, TU Braunschweig, 38106 Braunschweig, Germany

8 Deutsches Zentrum für Luft- und Raumfahrt (DLR), Institut für Planetenforschung, Rutherfordstraße 2, 12489 Berlin, Germany

9 Scientific Support Office, European Space Agency, 2201 Noordwijk, The Netherlands

10 Max-Planck-Institut für Sonnensystemforschung, Justus-vonLiebig-Weg, 3, 37077 Göttingen, Germany 
11 INAF-Osservatorio Astronomico, vicolo dell'Osservatorio 5, 35122 Padova, Italy

12 Aix-Marseille Université, CNRS, LAM, UMR 7326, 38 rue Frédéric Joliot-Curie, 13388 Marseille, France

13 International Space Science Institute, Hallerstraße 6, 3012 Bern, Switzerland

14 Centro de Astrobiología, CSIC-INTA, 28850 Torrejón de Ardoz, Madrid, Spain

15 Department of Physics and Astronomy, Uppsala University, Box 516, 75120 Uppsala, Sweden

16 PAS Space Research Center, Bartycka 18A, 00716 Warszawa, Poland

17 Department of Astronomy, University of Maryland, College Park, MD 20742-2421, USA

18 LESIA, Obs. de Paris, CNRS, Univ. Paris 06, Univ. Paris-Diderot, 5 place J. Janssen, 92195 Meudon, France

19 LATMOS, CNRS/UVSQ/IPSL, 11 boulevard d'Alembert, 78280 Guyancourt, France
20 Centro di Ateneo di Studi ed Attivitá Spaziali, "Giuseppe Colombo" (CISAS), University of Padova, 35131 Padova, Italy

21 CNR-IFN UOS Padova LUXOR, via Trasea, 7, 35131 Padova, Italy

${ }^{22}$ UNITN, Universitá di Trento, via Mesiano, 77, 38100 Trento, Italy

${ }^{23}$ Department of Mechanical Engineering - University of Padova, via Venezia 1, 35131 Padova, Italy

24 INAF-Osservatorio Astronomico, via Tiepolo 11, 34014 Trieste, Italy

25 Instituto de Astrofísica de Andalucía (CSIC), c/ Glorieta de la Astronomía s/n, 18008 Granada, Spain

26 National Central University, Graduate Institute of Astronomy, 300 Chung-Da Rd, 32054 Chung-Li, Taiwan

27 Laboratoire d'Astrophysique de Marseille, 38 rue Frédéric JoliotCurie, 13388 Marseille Cedex 13, France

28 Scientific Support Office, European Space Astronomy Centre/ESA, PO Box 78, 28691 Villanueva de la Canada, Madrid, Spain

${ }^{29}$ University of Padova, Department of Information Engineering, via Gradenigo 6/B, 35131 Padova, Italy 


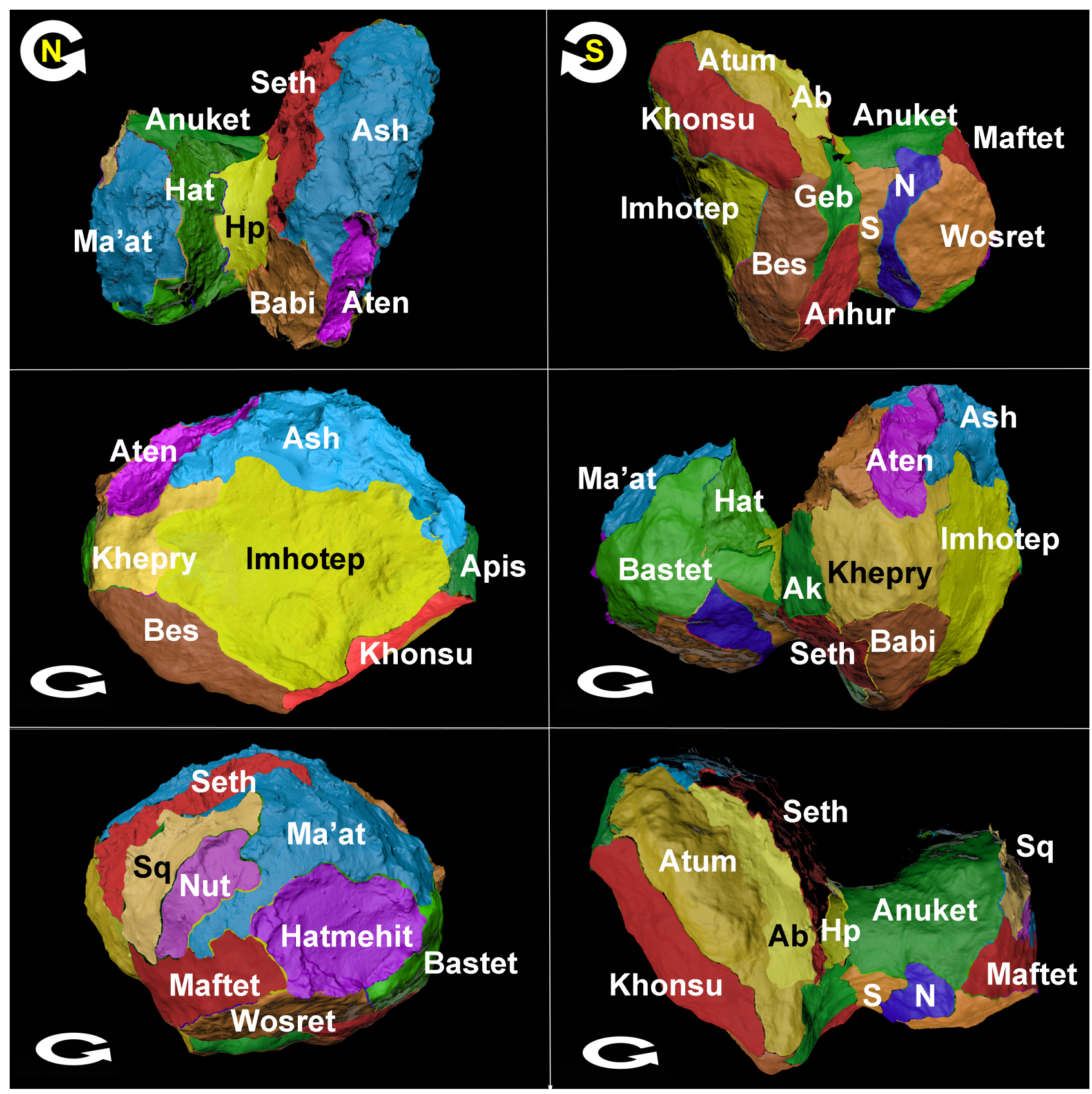

Fig. A.1. Six different viewing angles showing all the defined regions, including the northern hemisphere, on a shape model of the comet for global context and orientation. Acronyms used correspond to Hapi (Hp), Hathor (Hat) Sobek (S), Neith (N), Aker (Ak), and Serqet (Sq). Circular arrows show the direction of the comet's rotation. The two upper panels display the comet from a northern and southern polar viewing angles. The remaining four panels show the comet from an equatorial projection each with a shift of $\sim 90 \mathrm{deg}$. 
A\&A 593, A110 (2016)

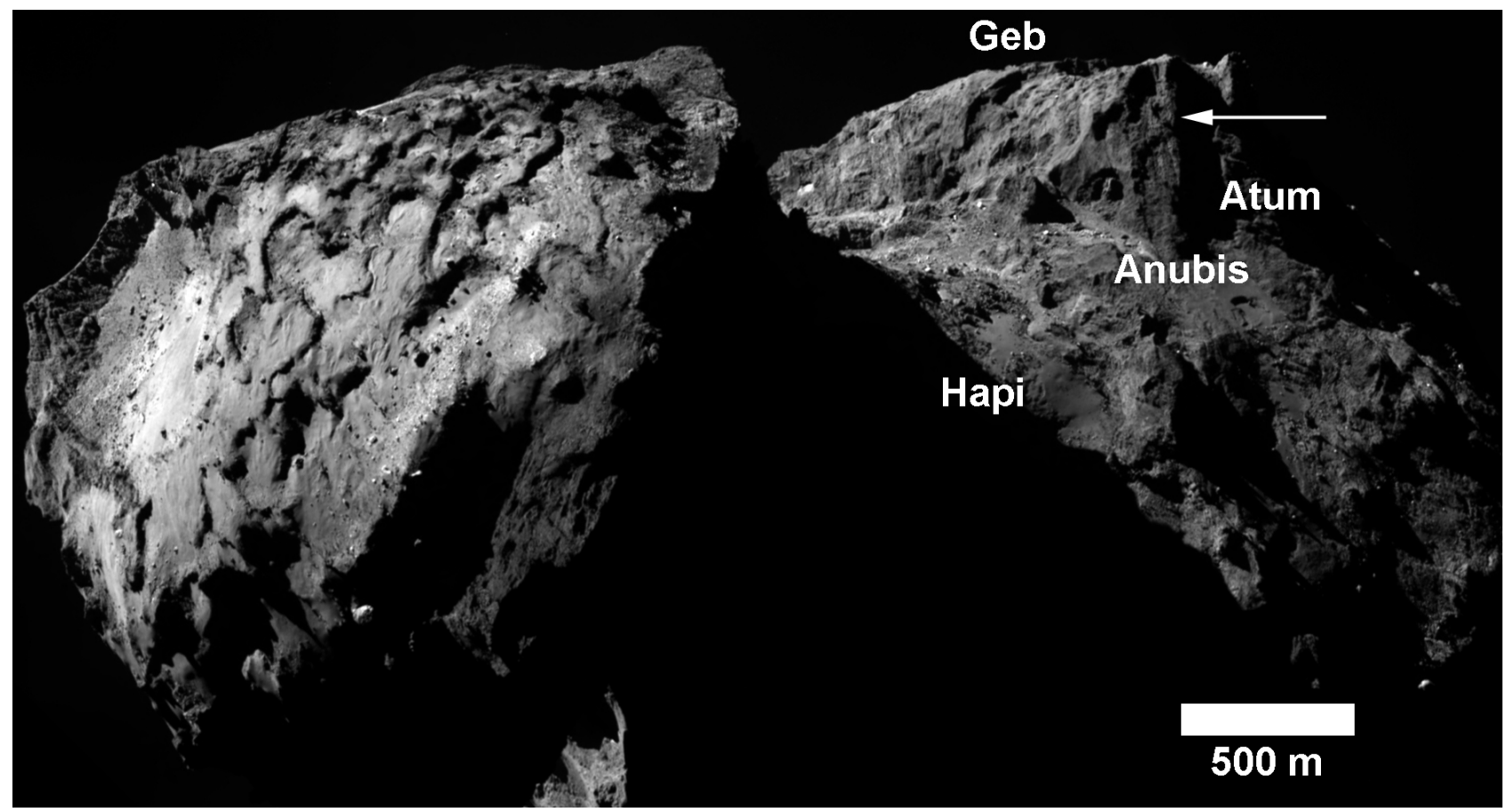

Fig. A.2. NAC image highlighting the boundary of Geb with the neighboring regions of Atum and Anubis. 
M. R. El-Maarry et al.: Regional morphology of comet 67P's southern hemisphere
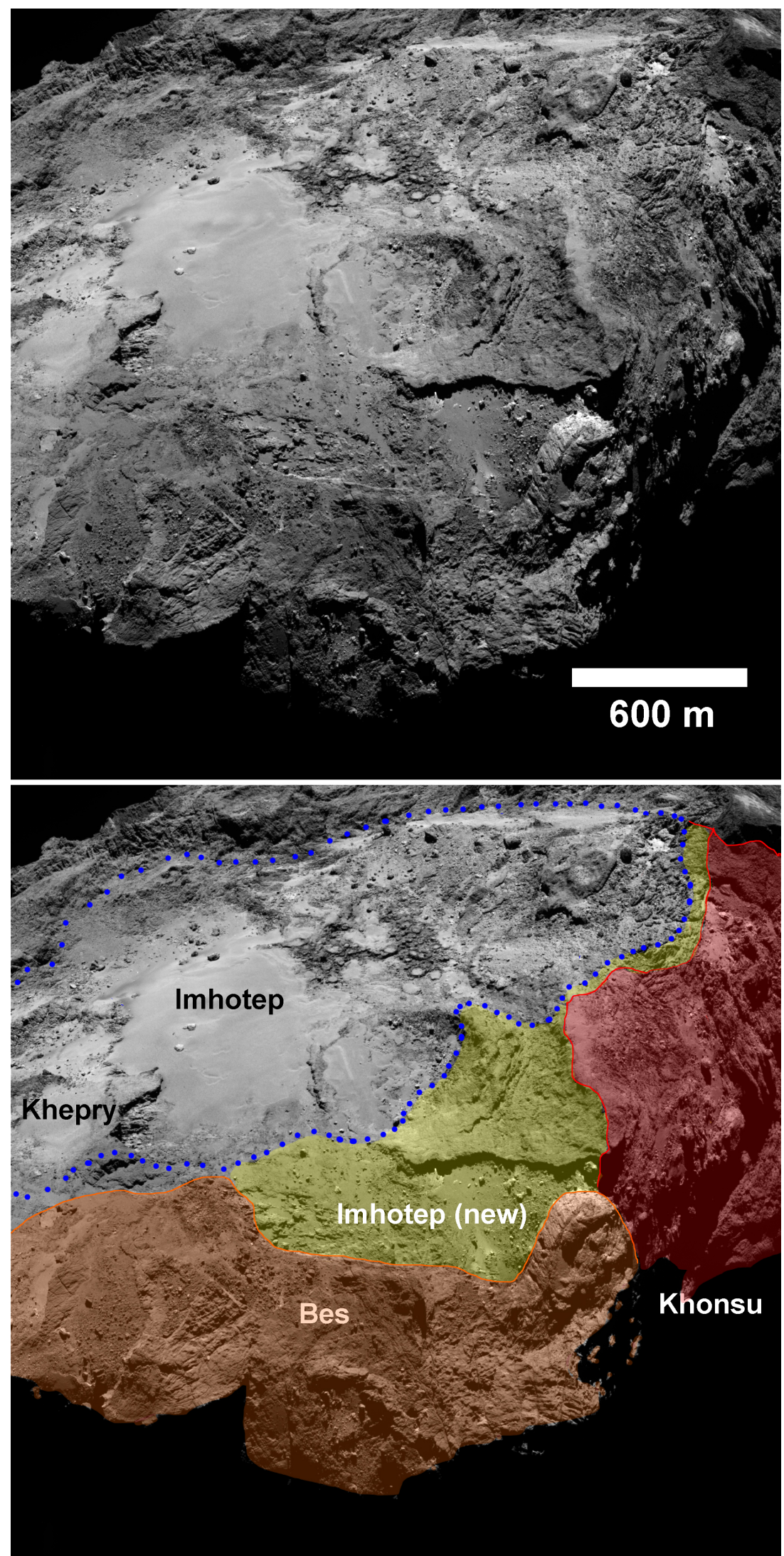

Fig. A.3. NAC image showing the Imhotep region and surrounding terrains along with a similar view with colored overlays of the regional boundaries and extent. The blue dotted line shows the old boundaries of Imhotep whereas the yellow region is the newly-added terrain. 


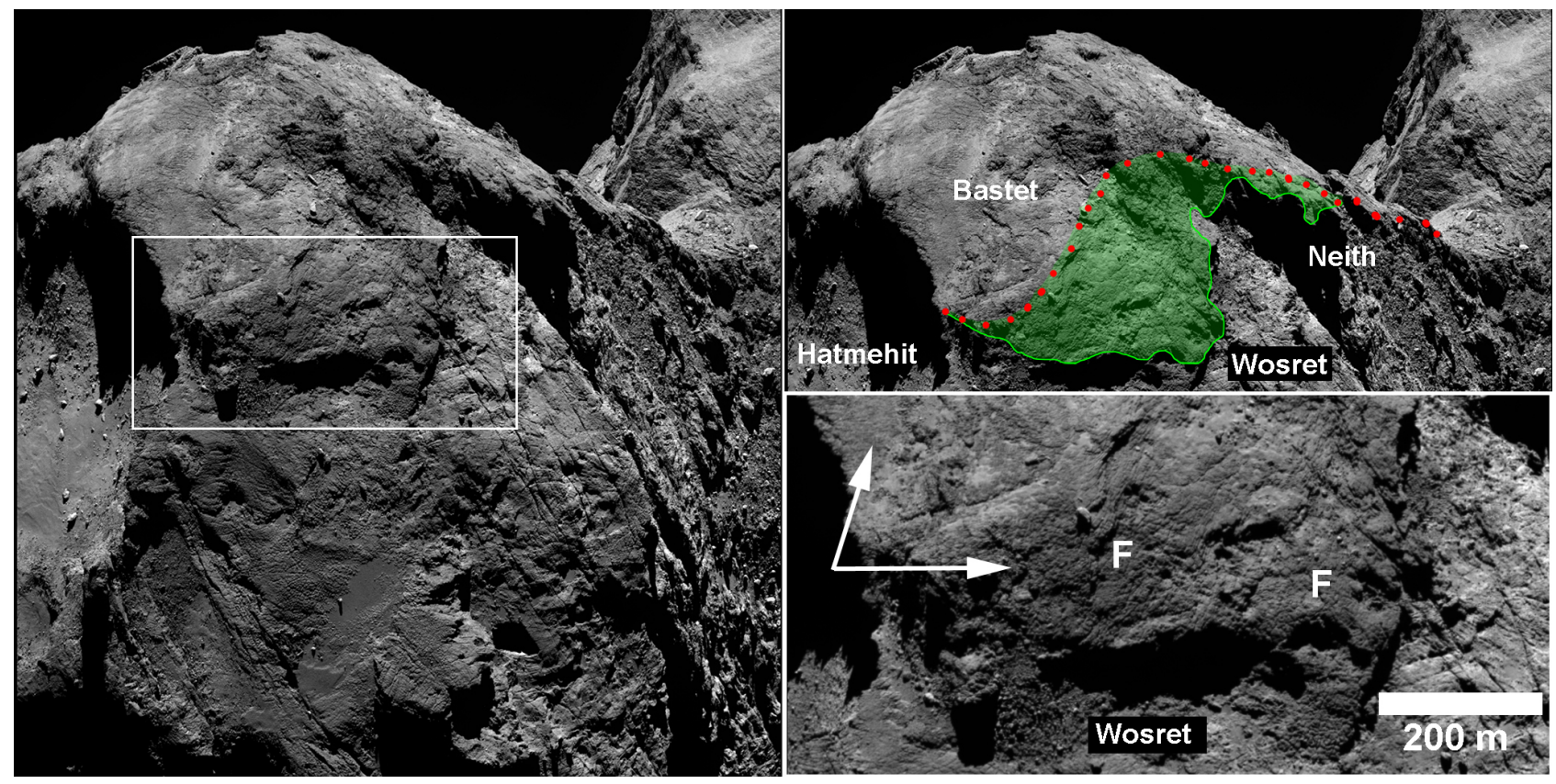

Fig. A.4. Left and top right: NAC image showing the Bastet region and surrounding terrains as well as a similar view with colored overlays of the regional boundaries. The red dotted line shows the old boundaries of Bastet whereas the green region is the newly-added terrain. The inbox shows the location highlighted in the bottom right panel. Bottom right: a full resolution view of the Bastet-Wosret boundary highlighting the fractured nature of the Bastet boundary (good examples highlighted by the letter "F"). Bastet forms a scarp overlying the Wosret region. Note the difference in tone between the northern portion of Bastet and the southern part (arrows). 


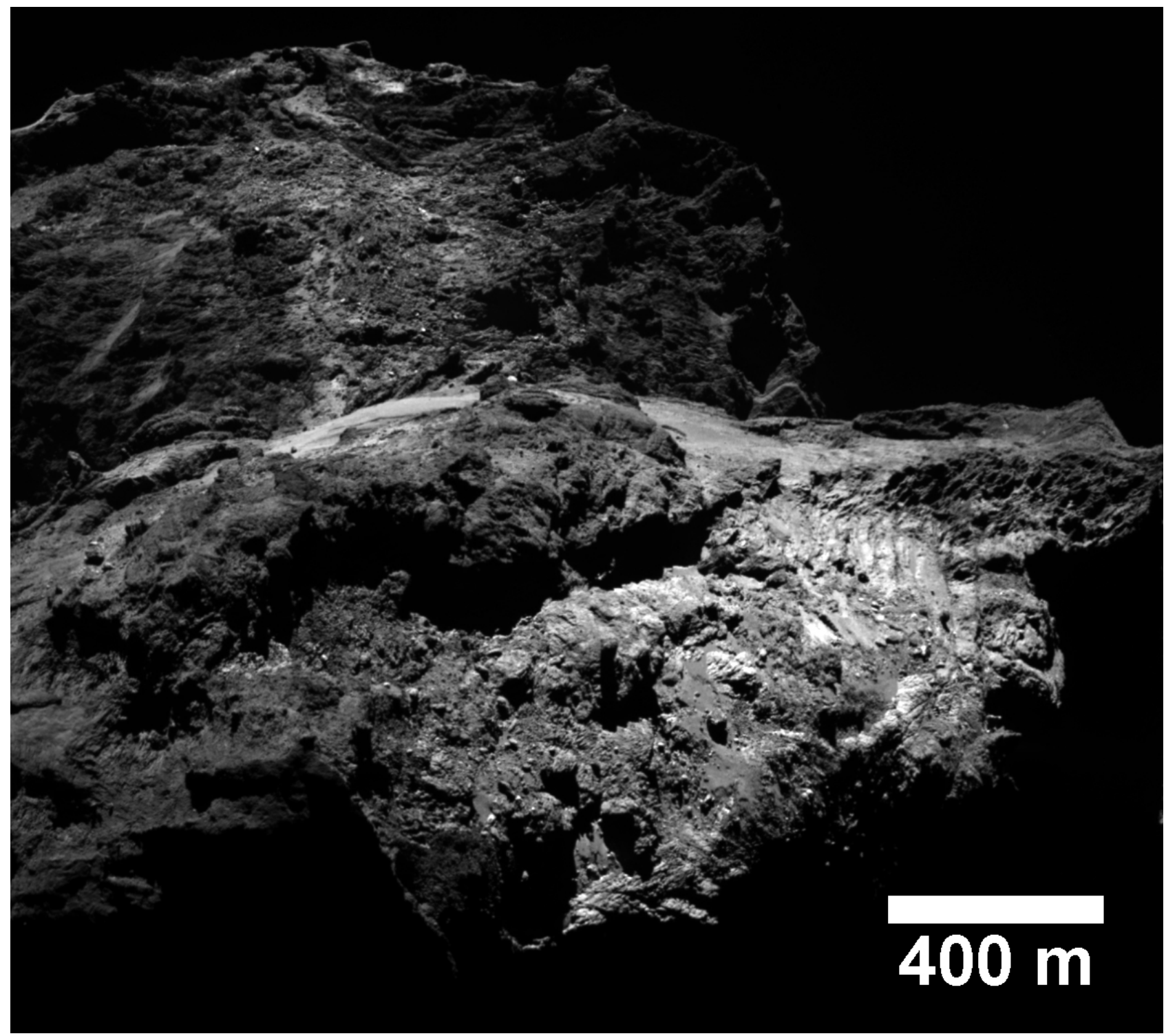

Fig. A.5. Part of a NAC image taken for the Khonsu region, which highlights its unusual shape and suggests that it may be a depression caused by a massive outburst in the past, and as a result, is peering into the internal structure of the comet. 
Table A.1. IDs for used images.

\begin{tabular}{|c|c|}
\hline Figure & Image ID \\
\hline 1 & $\begin{array}{l}\text { [From top to bottom]: } \\
\text { NAC_2015-07-29T19.29.45.737Z_IDB0_1397549500_F22 } \\
\text { NAC_2015-05-02T10.42.41.352Z_ID30_1397549001_F22 } \\
\text { NAC_2015-05-02T15.09.31.384Z_ID30_1397549001_F22 }\end{array}$ \\
\hline 2 & NAC_2016-01-27T16.27.58.970Z_ID10_1397549500_F22 \\
\hline 3 & NAC_2016-01-27T11.53.41.927Z_ID10_1397549000_F22 \\
\hline 4 & $\begin{array}{l}\text { [Left]: NAC_2016-01-23T22.45.22.132Z_IDB0_1397549001_F22 } \\
\text { [Right]: NAC_2016-01-27T11.53.41.927Z_ID10_1397549000_F22 }\end{array}$ \\
\hline 5 & $\begin{array}{c}\text { [Left]: NAC_2016-01-30T07.58.39.713Z_IDB0_1397549800_F22 } \\
\text { [Top right]: NAC_2015-12-07T14.02.55.651Z_IDB0_1397549000_F22 } \\
\text { [Bottom right]: NAC_2016-01-23T17.03.47.168Z_IDB0_1397549001_F22 }\end{array}$ \\
\hline 6 & Digital terrain model adapted from the shape model SHAP5 \\
\hline 7 & $\begin{array}{c}\text { [Top]: NAC_2016-01-27T17.20.08.041Z_ID10_1397549000_F22 } \\
\text { [Bottom]: NAC_2016-02-07T10.18.08.701Z_ID10_1397549500_F22 } \\
\text { [Inbox]: NAC_2015-12-12T16.10.02.283Z_ID30_1397549003_F22 }\end{array}$ \\
\hline 8 & NAC_2016-02-07T07.27.51.515Z_IDB0_1397549004_F22 \\
\hline 9 & $\begin{array}{l}\text { [Top]: NAC_2016-01-27T07.44.41.724Z_ID10_1397549800_F22 } \\
\text { [Bottom]: NAC_2016-02-07T08.11.51.516Z_IDB0_1397549008_F22 }\end{array}$ \\
\hline 10 & NAC_2016-02-10T19.20.22.115Z_IDB0_1397549800_F22 \\
\hline 11 & Cylindrical map projection from the SHAP5 shape model \\
\hline A.1 & $\begin{array}{l}\text { [From top to bottom]: } \\
\text { WAC_2014-09-05T02.29.12.717Z_ID30_1397549700_F18 } \\
\text { NAC_2014-08-16T13.59.14.564Z_ID30_1397549600_F22 } \\
\text { NAC_2014-08-05T23.19.14.571Z_ID30_1397549800_F22 } \\
\text { NAC_2014-08-16T13.59.14.564Z_ID30_1397549600_F22 }\end{array}$ \\
\hline A. 2 & NAC_2015-11-28T21.46.25.980Z_IDB0_1397549005_F22 \\
\hline A.3 & NAC_2016-01-27T14.01.32.942Z_ID10_1397549700_F22 \\
\hline A. 4 & NAC_2016-02-10T07.06.04.905Z_IDB0_1397549400_F22 \\
\hline A.5 & NAC_2015-12-18T03.43.20.332Z_IDB0_1397549001_F22 \\
\hline
\end{tabular}

Notes. The first three letters show the instrument used to acquire the image (All are NAC). The following 15 digits display the time (in UTC) of imaging in year-month-day format followed by hour-min-sec. Finally, the last two numbers correspond to the filters used (Keller et al. 2007). All images used here are for the "orange" filter. Figure A.1 is adapted from El-Maarry et al. (2015a). 